\title{
Pole Distribution of PVI Transcendents close to a Critical Point (summer 2011)
}

\author{
Davide Guzzetti \\ SISSA, Intenational School of Advanced Studies, 34136 Trieste, Italy \\ and KIAS, Korea Institute of Advanced Study, 130-722 Seoul, South Korea \\ Tel +82 -2-958-3861 \\ davide_guzzetti@yahoo.com
}

\begin{abstract}
The distribution of the poles of Painlevé VI transcendents associated to semi-simple Frobenius manifolds is determined close to a critical point. It is shown that the poles accumulate at the critical point, asymptotically along two rays. As an example, the Frobenius manifold given by the quantum cohomology of $C P^{2}$ is considered. The general PVI is also considered.
\end{abstract}

MSC: 34M55 (Painlevé and other special functions)

\section{Introduction}

Consider the Sixth Painlevé equation associated to a three dimensional Frobenius manifold, hereafter denoted $P V I_{\mu}$, with parameters $\alpha=\frac{(2 \mu-1)^{2}}{2}, \beta=\gamma=0, \delta=\frac{1}{2}$ (in standard notation).

$$
\begin{gathered}
\frac{d^{2} y}{d x^{2}}=\frac{1}{2}\left[\frac{1}{y}+\frac{1}{y-1}+\frac{1}{y-x}\right]\left(\frac{d y}{d x}\right)^{2}-\left[\frac{1}{x}+\frac{1}{x-1}+\frac{1}{y-x}\right] \frac{d y}{d x} \\
+\frac{1}{2} \frac{y(y-1)(y-x)}{x^{2}(x-1)^{2}}\left[(2 \mu-1)^{2}+\frac{x(x-1)}{(y-x)^{2}}\right], \quad \mu \in \mathbf{C .} \quad\left(P V I_{\mu}\right)
\end{gathered}
$$

The algebraic solutions of $P V I_{\mu}$ were studied in [6], its elliptic representation in [10]. The importance of $P V I_{\mu}$ in the theory of semi-simple Frobenius manifolds was extabilished in [4] and [5], and practically applied in [9] to the construction of some relevant manifolds.

In this paper, we study the distribution of the movable poles of Painlevé transcendents, close to the critical point $x=0$. We do this for any $\mu \in \mathbf{C}$. Due to the symmetries of $P V I_{\mu}$, the results, obtained close to $x=0$, can be translated to the poles close to $x=1$ and $x=\infty$. We shown that the poles accumulate at the critical point, asymptotically along two rays. The results can be extended to the general Painlevé VI equation, as sketched in section 5.

The distribution of the poles close to a critical point for $P V I_{\mu}$ has been anticipated in [10], where it was conjectured that the poles of a transcendent, considered as the meromorphic extension of a branch on the universal covering of the critical point, should accumulate at the critical point along spirals. The same conjecture is motivated in [11] for the general Painlevé VI equation. In [1], the pole distribution for PVI with parameters $\alpha=\beta=\gamma=1 / 8$ and $\delta=3 / 8$ (Hitchin's equation [16]) is determined on the whole universal covering of $\mathbf{C} \backslash\{0,1, \infty\}$. A formula for an infinite series of poles is given in terms of Theta-functions. The poles are distributed along lines which are spirals at a small scale around the critical points, and more complicated lines on the whole universal covering. A birational Okamoto's transformation 
transforms the above PVI equation into $P V I_{\mu}$ with $\mu=1 / 2$. The latter, studied by Picard [24], will be considered in section 4 .

In this paper, we determine the poles of Painlevé transcendents in a neighborhood of $x=0$, with bounded $\arg x$, namely $|\arg x|<\vartheta$, for some $\vartheta>0$. Thus, $x$ may tend to zero along a radial path, while spiral paths are not allowed. With this limitation, "most" solutions of PVI have no poles in a sufficiently small neighborhood of the critical point, except for a class of solutions, which is the object of this paper. Actually, the critical behaviors at one of the critical points $x=0,1$ or $\infty$ of a branch $y(x)$ can be divided into a few classes, classified in [14] and [15] (an equivalent classification is given in [2] [3]). Among them, one class of three-real parameters solutions may admit poles in a neighborhood of the critical point, with bounded $\arg x$. The class is given in Proposition 1 of [14] for the general Painlevé VI equation. In the particlular case of $P V I_{\mu}$, the proposition states the following:

Let $\nu \in \mathbf{R}, \nu \neq 0$, and $d \in \mathbf{C}$ be given, such that $2 \mu-1 \neq \pm 2 i \nu$. Let also $\vartheta>0$ be given. Equation $P V I_{\mu}$ has a solution $y(x)$ admitting the following expansion when $x \rightarrow 0$ and $|\arg x|<\vartheta$ :

$$
\frac{1}{y(x)}=y_{1}(x)+x y_{2}(x)+x^{2} y_{3}(x)+\ldots=\sum_{n=1}^{\infty} x^{n-1} y_{n}(x)
$$

where:

$$
y_{n}(x)=\sum_{m=-n}^{n} A_{n m}(\nu, \mu) e^{2 i m d} x^{2 i m \nu}
$$

For any $\vartheta$, there exists a sufficiently small $\epsilon$ such that the series of $1 / y(x)$ converges in the domain $0<|x|<\epsilon,|\arg x|<\vartheta$, and defines an holomorphic function of $x$ and $x^{2 i \nu}$. The $A_{n m}(\nu, \mu)$ 's are rational functions of $\nu, \mu$, and satisfy the property $\bar{A}_{n, m}(\nu, \mu)=A_{n,-m}(\nu, \mu)=A_{n m}(-\nu, \mu)$ (the bar denotes the complex conjugate). Their explicit form is recursively computed by the procedure of [14]. For example, the lower order coefficients are:

$$
A_{11}=\frac{(2 \mu-1-2 i \nu)^{2}}{16 \nu^{2}}, \quad A_{10}=-\frac{(2 \mu-1)^{2}-4 \nu^{2}}{8 \nu^{2}} .
$$

and the first order approximation is:

$$
\begin{gathered}
y_{1}(x)=\frac{(2 \mu-1+2 i \nu)^{2} e^{-2 i d} x^{-2 i \nu}}{16 \nu^{2}}-\frac{(2 \mu-1)^{2}-4 \nu^{2}}{8 \nu^{2}}+\frac{(2 \mu-1-2 i \nu)^{2} e^{2 i d} x^{2 i \nu}}{16 \nu^{2}} \\
\equiv 1-\frac{4 \nu^{2}+(2 \mu-1)^{2}}{4 \nu^{2}} \sin ^{2}\left(\nu \ln x+d+\frac{i}{2} \ln \left[\frac{2 \mu-1+2 i \nu}{2 \mu-1-2 i \nu}\right]\right)
\end{gathered}
$$

The second order coefficients are:

$$
\begin{gathered}
A_{22}=-\frac{(-2 \mu+2 i \nu+1)^{4}}{2^{9} \nu^{4}}, \quad A_{21}=\frac{\left((2 \mu-1)^{2}+8 i \nu^{3}\right)(-2 \mu+2 i \nu+1)^{2}}{2^{7} \nu^{4}}, \\
A_{20}=-\frac{\left((2 \mu-1)^{2}+4 \nu^{2}\right)\left(3\left(2 \mu^{2}-1\right)^{2}-4 \nu^{4}\right)}{2^{8} \nu^{4}} .
\end{gathered}
$$

The other coefficients up to order $y_{4}$ are in the Appendix of the preprint version arXiv:1104.5066. Due to the structure of $y_{n}(x)$, which is invariant for $\nu \mapsto-\nu$ and $d \mapsto d+k \pi, k$ integer, we are allowed to assume that

$$
0 \leq \Re d \leq \pi, \quad \nu>0
$$


We remark that (1) is derived in [14] by a symmetry transformation applied to a solution of a PVI equation with $\alpha=\gamma=1-2 \delta=0$ and $\beta \neq 0$, defined for $\arg x$ bounded, namely $|\arg x|<\vartheta$, for some $\vartheta>0$. The latter solution can be locally constructed both by the method of [19] or the method of local analysis of [26] (see also [18]) and [11]. It follows from these methods that $\vartheta$ is chosen finite but arbitrarily, observing that, if $\vartheta$ is increased, the radius of convergence of (1) in general decreases.

\subsection{Poles close to the Critical Point and main Results of the Paper}

Clearly, the $n$-th order $y_{n}(x)$ is an oscillatory bounded function in a neighborhood of $x=0$. The leading term of $1 / y(x)$ is then $y_{1}(x)$, while $x^{n-1} y_{n}(x)=O\left(x^{n-1}\right)$. The solution $y(x)$ with expansion (1) may have poles in a neighborhood of $x=0$, which are the zeros of $\sum_{n=1}^{\infty} x^{n-1} y_{n}(x)$. The zeros of $y_{1}(x)$ do not coincide in general with the zeros of $\sum_{n=1}^{\infty} x^{n-1} y_{n}(x)$. Thus, one cannot write:

$$
y(x)=\frac{1}{y_{1}(x)}\left[1-x \frac{y_{2}(x)}{y_{1}(x)}+O\left(x^{2}\right)\right], \quad x \rightarrow 0
$$

The above asymptotic expansion is true only when $x \rightarrow 0$ in a sector not containing the zeros of both $y_{1}(x)$ and $\sum_{n=1}^{\infty} x^{n-1} y_{n}(x)$ (in this case, the leading term $1 / y_{1}(x)$ of the expansion is computed also in [2] and [3]). We expect that the poles of $y(x)$ are close to the zeros of $y_{1}(x)$ as $x \rightarrow 0$, because $y(x)^{-1} \sim y_{1}(x)$. It is to be remarked that any considerations about the poles of $y(x)$ must be done for $|x|$ smaller than the radius of convergence of the series (1).

The general result of the paper is summarized in the following theorem.

Theorem 1 Let $\nu>0$ and $d \in \mathbf{C}, 0 \leq \Re d \leq \pi$, be given such that $2 \mu-1 \neq \pm 2 i \nu$, and let $y(x)$ be (1). Then, $y_{1}(x)$ has the two sequences of zeros

$$
x_{k}(1)=\exp \left\{-\frac{d}{\nu}-\frac{k \pi}{\nu}\right\}=\exp \left\{-i \frac{\Im d}{\nu}\right\} \exp \left\{-\frac{\Re d}{\nu}-\frac{k \pi}{\nu}\right\}, \quad k \in \mathbf{Z},
$$

and

$$
\begin{aligned}
x_{k}(2) & =x_{k}(1) \exp \left\{-\frac{i}{\nu} \ln \left(\frac{2 \mu-1+2 i \nu}{2 \mu-1-2 i \nu}\right)\right\}= \\
& =x_{k}(1) \exp \left\{\frac{1}{\nu} \arg \left(\frac{2 \mu-1+2 i \nu}{2 \mu-1-2 i \nu}\right)\right\} \exp \left\{-\frac{i}{\nu} \ln \left|\frac{2 \mu-1+2 i \nu}{2 \mu-1-2 i \nu}\right|\right\} .
\end{aligned}
$$

where $-\pi<\arg \left(\frac{2 \mu-1+2 i \nu}{2 \mu-1-2 i \nu}\right) \leq \pi$, being other choices absorbed into $\frac{k \pi}{\nu}$. Let $k_{0} \in \mathbf{N}$ be sufficiently big in order for $x_{k}(j), j=1,2$ to fall in the domain of convergence of the series (1). There exists $K$ sufficientley big such that for every $k \geq \max \left\{K, k_{0}\right\}$, and every $j=1,2, y(x)$ has a pole $\xi_{k}(j)$ lying in a neighborhood of $x_{k}(j)$, with the following asymptotic representation

$$
\xi_{k}(j)=x_{k}(j)-\frac{1}{2} x_{k}(j)^{2}+\sum_{N=3}^{\infty} \Delta_{N}(j) x_{k}(j)^{N}, \quad k \rightarrow+\infty, \quad x_{k}(j) \rightarrow 0 .
$$

The coefficients $\Delta_{N}(j) \in \mathbf{C}$ are certain numbers independent of $k$ that can be computed form the coefficients $A_{n m}$ of (2). The first terms are:

$$
\begin{gathered}
\Delta_{3}(1)=\frac{16 \mu^{4}-8 \mu^{2}+176 \nu^{4}+352 \nu^{2}+177}{1024\left(\nu^{2}+1\right)^{2}} \\
\Delta_{3}(2)=\frac{16 \mu^{4}-64 \mu^{3}+88 \mu^{2}-48 \mu+176 \nu^{4}+352 \nu^{2}+185}{1024\left(\nu^{2}+1\right)^{2}}
\end{gathered}
$$




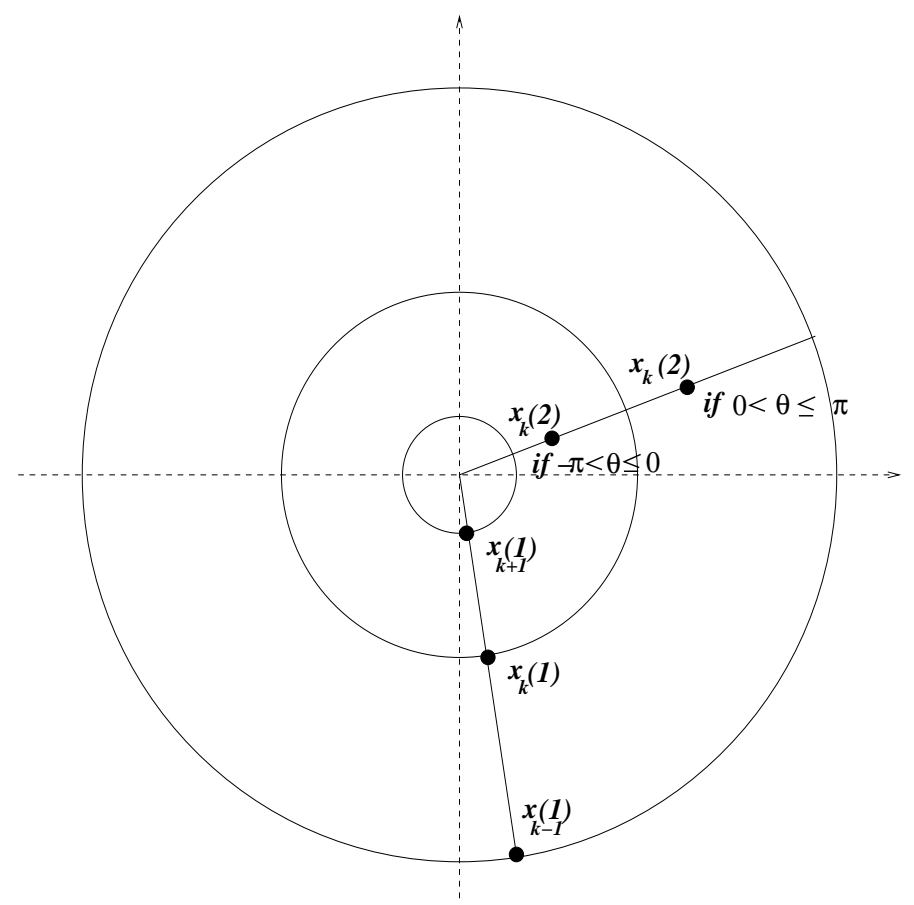

Figure 1: Position of the zeros of $y_{1}(x) . \quad \theta=\arg \left(\frac{2 \mu-1+2 i \nu}{2 \mu-1-2 i \nu}\right)$. Observe that $\left|x_{k+1}(1)\right|<$ $\left|x_{k}(2)\right| \leq\left|x_{k}(1)\right|$ when $-\pi<\arg \left(\frac{2 \mu-1+2 i \nu}{2 \mu-1-2 i \nu}\right) \leq 0$, and $\left|x_{k}(1)\right|<\left|x_{k}(2)\right| \leq\left|x_{k-1}(1)\right|$ when $0<\arg \left(\frac{2 \mu-1+2 i \nu}{2 \mu-1-2 i \nu}\right) \leq \pi$.

$$
\begin{gathered}
\Delta_{4}(1)=-\frac{16 \mu^{4}-8 \mu^{2}+49+48 \nu^{4}+96 \nu^{2}}{1024\left(\nu^{2}+1\right)^{2}} \\
\Delta_{4}(2)=-\frac{16 \mu^{4}-64 \mu^{3}+88 \mu^{2}-48 \mu+57+48 \nu^{4}+96 \nu^{2}}{1024\left(\nu^{2}+1\right)^{2}}
\end{gathered}
$$

In the following, when the $x_{k}(j), \mathrm{j}=1,2$, are considered, it will be understood that $k \geq k_{0}$. When $k \rightarrow+\infty$, the zeros $x_{k}(1)$ accumulate at $x=0$ along the ray of angle $-\frac{\Im d}{\nu}$, while the zeros $x_{k}(2)$ accumulate at $x=0$ along the ray of angle $-\frac{1}{\nu}\left(\Im d+\ln \left|\frac{2 \mu-1+2 i \nu}{2 \mu-1-2 i \nu}\right|\right)$. A typical case is that of figure 1 . For real $\mu$ the two rays coincide. If $\mu=1 / 2$, then $\Delta_{N}(1)=\Delta_{N}(2)$ and the poles of the two sequences overlap (double poles). The series (5) is at least asymptotic, but we can prove its convergence when $\mu=\frac{1}{2}$ (section 4). Observe that in order for the rays of the zeros to fall into the domain where the series (1) is defined, $\vartheta$ must be sufficiently big (see also the remark at the end of section 2). .

Remark: Each pole $\xi_{k}(j)$ lies in a disk centered in $x_{k}(j)$ of radius $\frac{1}{2}\left[x_{k}(j)\right]^{2}+O\left(\left[x_{k}(j)\right]^{3}\right)$, as depicted in figure 2. The zeros are ordered as $\left|x_{k+1}(j)\right|<\left|x_{k}(l)\right|<\left|x_{k}(j)\right|$, where $(j, l)=(1,2)$ or $(2,1)$. Consider disks centered at these zeros of radius $\left|x_{k}(j)\right|^{2},\left|x_{k}(l)\right|^{2},\left|x_{k+1}(j)\right|^{2}$. $K$ is constructed in the proof of the theorem in such a way that when $k \geq K$ the disks do not intersect. Thus, Theorem 1 is consistent, because $\xi_{k}(j)$ is closer to $x_{k}(j)$ than to any other $x_{k^{\prime}}\left(j^{\prime}\right)$.

We may ask if there are other poles of $y(x)$, in the domain of convergence of (1), other than 


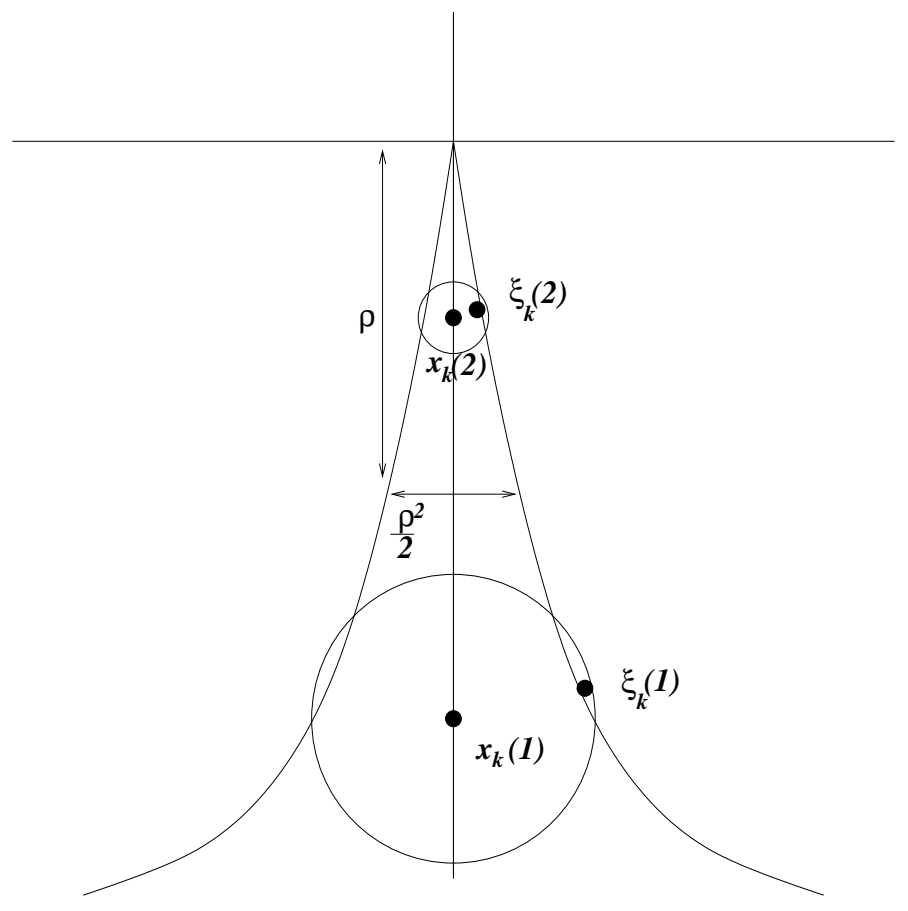

Figure 2: The disks around the zeros $x_{k}(j)$ of $y_{1}(x)$, where the poles $\xi_{k}(j)$ of $y(x)$ possibly lie. The figure represents the case $\mu=-1, \nu=2 \ln \mathbf{G} / \pi, \mathbf{G}=(1+\sqrt{5}) / 2$. In this case, the two rays coincide with the negative imaginary axis.

those of Theorem 1 . Let $R<$ radius of convergence of $(1)<1$ be the radial coordinate for a point on the ray where the zeros of $y_{1}(x)$ lie. We prove that $y(x)$ has no poles that are more than $R^{2}$-distant from the rays, as in figure 2 . To formalize this statement, let $\mathcal{U}(R, \epsilon)$ be a close domain constructed by taking a disk centered at $x=0$ of radius $R$, minus two sectors bisected by the rays where the zeros lie. Each sector has angular amplitude $2 \epsilon$. See figure 3 , where the general case and the case $\mu=-1$ are depicted.

Theorem 2 Let $y(x)$ be (1). For any small $\epsilon>0$, there exist $R_{\epsilon}<$ radius of convergence of (1), such that $y(x)$ has no poles in $\mathcal{U}\left(R_{\epsilon}, \epsilon\right)$. When $\epsilon \rightarrow 0, R_{\epsilon}$ can be chosen to be any number such that:

$$
\begin{array}{rr}
R_{\epsilon}<\frac{|2 \mu-1|}{C_{f}} \tan \epsilon\left(1+O\left(\tan ^{2} \epsilon\right)\right), & \mu \neq \frac{1}{2} . \\
R_{\epsilon}<\frac{2 \nu^{2}}{C_{f}} \tan ^{2}(\epsilon)\left(1+O\left(\tan ^{3}(\epsilon)\right)\right), & \mu=\frac{1}{2} .
\end{array}
$$

The asymptotic estimate $R_{\epsilon} \sim \frac{|2 \mu-1| \tan \epsilon}{C_{f}}$ for $\epsilon \rightarrow 0$ means that the poles of $y(x)$, if they exist, get closer to the the rays where the zeros lie, as their absolute value decreases. Their distance from the the rays is at most $|2 \mu-1| \epsilon \tan \epsilon / C_{f} \cong|2 \mu-1| \epsilon^{2} / C_{f} \cong C_{f} R_{\epsilon}^{2} /|2 \mu-1|$ (or $\sqrt{C_{f}} R_{\epsilon}^{\frac{3}{2}} / \sqrt{2} \nu$ if $\left.\mu=\frac{1}{2}\right)$.

A relevant example of Frobenius manifold is given by the quantum cohomology of the two dimensional complex projective space $C P^{2}$. A solution of $P V I_{\mu}$, with $\mu=-1$, is associated to 

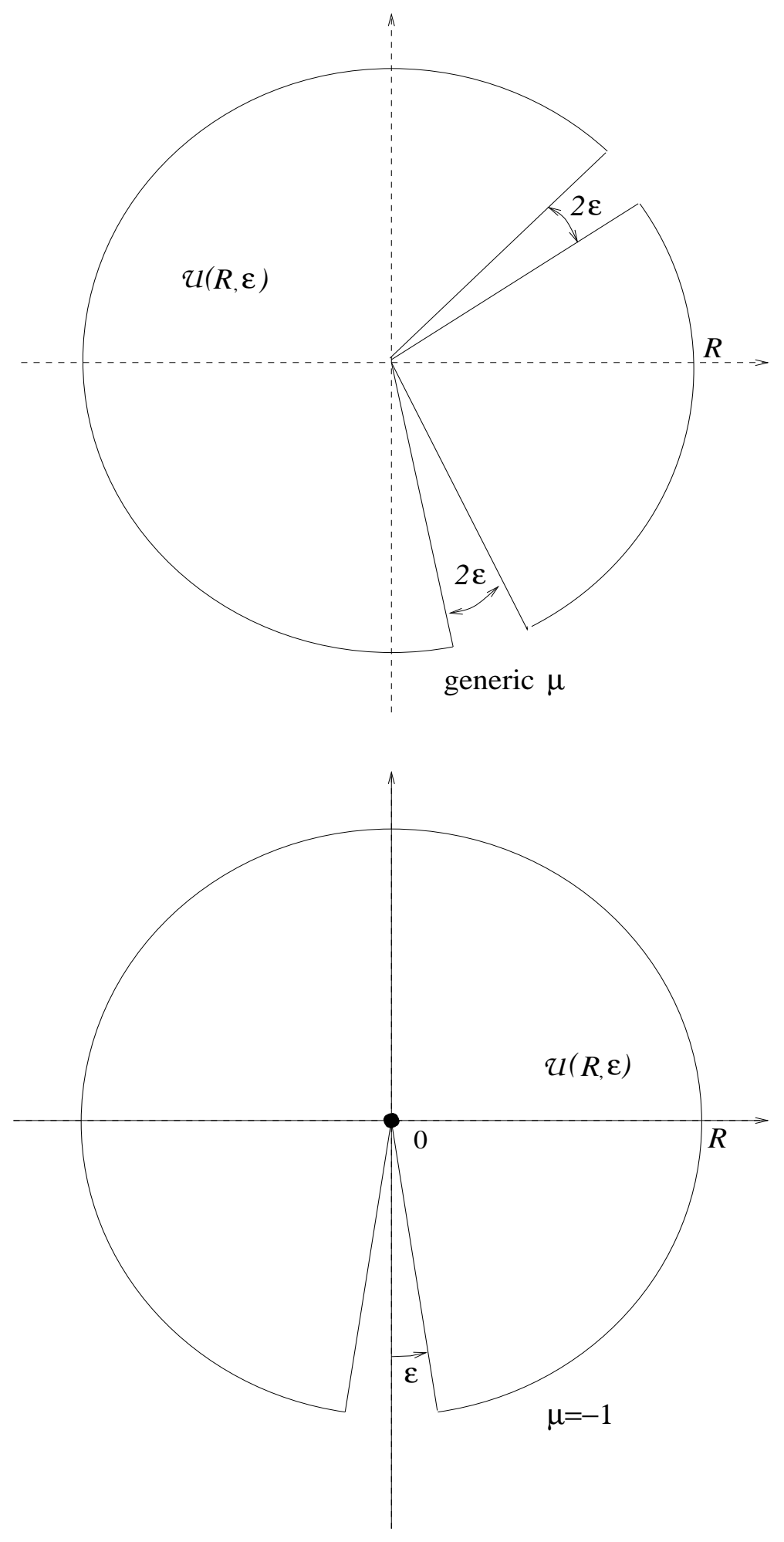

Figure 3: 
this manifold [5]. It is a solution of the form (1) (please, see section 2 in order to understand this fact). In this case, we prove that

$$
\nu=\frac{2 \ln \mathbf{G}}{\pi}, \quad \mathbf{G}=\frac{1+\sqrt{5}}{2}
$$

where $\mathbf{G}$ is the golden ratio. This very special value makes the two rays of zeros coincide with the negative immaginary axis. Note that we can choose $\vartheta=\pi$, in such a way that (1) becomes a branch with branch cut along the negative axis. The result is summarized in the following theorem:

Theorem 3 The branch (1) associated to the Quantum Cohomology of $C P^{2}$, defined for $|\arg x|<$ $\pi$, which satisfies the equation $P V I_{\mu=-1}$, is identified by the following integration contants:

$$
\begin{gathered}
\nu=\frac{2 \ln \mathbf{G}}{\pi}=0.30634 \ldots \\
d=\frac{i}{2} \ln \left\{-\pi^{2} \frac{\left(\mathbf{G}^{4}+1\right)^{2}}{\left(\mathbf{G}^{2}+1\right)^{2}} 2^{16 i \nu} \frac{(1-2 i \nu)^{2} \nu^{2}}{(1+2 i \nu)^{2}} \frac{\Gamma(1-2 i \nu)^{4}}{\Gamma(1-i \nu)^{8}}\right\},
\end{gathered}
$$

The first approximation $y_{1}(x)$ has two infinite sequences of zeros accumulating at $x=0$ along the negative imaginary axis:

$$
x_{k}(j)=-i \exp \left\{-\frac{\Re d}{\nu}-\frac{2(j-1)}{\nu}\left|\arccos \frac{3}{\sqrt{4 \nu^{2}+9}}\right|\right\} \exp \left\{-\frac{k \pi}{\nu}\right\}, \quad k \in \mathbf{N}, \quad j=1,2 .
$$

The branch (1) has two sequences of poles $\left\{\xi_{k}(1)\right\}_{k \geq k_{0}},\left\{\xi_{k}(2)\right\}_{k \geq k_{0}}$ in a neighborhood of $x=0$, which accumulate at $x=0$ as $k \rightarrow \infty$, asymptotically approaching the negative imaginary axis, according to the asymptotic expansion (5), where the first terms are:

$$
\begin{gathered}
\Delta_{3}(1)=\frac{176 \nu^{4}+185+352 \nu^{2}}{1024\left(\nu^{2}+1\right)^{2}}=0.1792 \ldots, \quad \Delta_{3}(2)=\frac{401+176 \nu^{4}+352 \nu^{2}}{1024\left(\nu^{2}+1\right)^{2}}=0.3555 \ldots \\
\Delta_{4}(1)=-\frac{57+48 \nu^{4}+96 \nu^{2}}{1024\left(\nu^{2}+1\right)^{2}}=-0.05422 \ldots, \quad \Delta_{4}(2)=-\frac{\left.273+48 \nu^{4}+96 \nu^{2}\right)}{1024\left(\nu^{2}+1\right)^{2}}=-0.2305 \ldots
\end{gathered}
$$

Corollary: $d$ of Theorem 3 has the following series

$$
d=\frac{\pi}{2}-8 \nu \ln (2)+2 \arccos \frac{1}{\sqrt{1+4 \nu^{2}}}+4 \sum_{n=1}^{\infty} \frac{(-1)^{n}\left(1-4^{n}\right) \zeta(2 n+1)}{2 n+1} \nu^{2 n+1}+i \frac{\pi \nu}{2}+k \pi
$$

where $2 \arccos \left(1 / \sqrt{1+4 \nu^{2}}\right)=2 \sum_{n=0}^{\infty}(-1)^{n}(2 \nu)^{2 n+1}$, and $k \in \mathbf{Z}$. The above series is absolutely convergent for $|\nu|<\frac{1}{2}$, therefore well defined for $\nu=\frac{2}{\pi} \ln \mathbf{G}$. In particular, $\Im d=\frac{\pi \nu}{2}$.

According to (4), we take $k=0$ in the corollary. The first terms of the series then give the numerical value $\Re d=1.08323 \ldots$ and $\frac{\Re d}{\nu}=3.53595 \ldots$. Other numerical values are: $\arg \left(\frac{3-2 i \nu}{3+2 i \nu}\right)=$ $-2 \operatorname{arcos}\left(\frac{3}{\sqrt{4 \nu^{2}+9}}\right)=-0.402 \ldots$, therefore $e^{\frac{1}{\nu} \arg \left(\frac{3-2 i \nu}{3+2 i \nu}\right)}=0.268 \ldots, e^{-\frac{\Re d}{\nu}}=0.0291 \ldots, e^{-\frac{\pi}{\nu}}=$ $3.52 \ldots \times 10^{-5}$. It follows that the first two zeros for $k \geq 1$ are $x_{0}(1)=-2.91 \ldots \times 10^{-2} i$ and $x_{0}(2)=-7.82 \ldots \times 10^{-3} i$. 
In Section 2, we review the dependence of the integration constants $d$ and $\nu$ on the monodromy data associated to a solution of $P V I_{\mu}$, for any $\mu$. It turns out that $d$ depends explicitly on $\nu$. In Section 3 we expand $d$ as a convergent Taylor series of $\nu$ :

$$
d=\sum_{n=1}^{\infty} d_{n} \nu^{n}, \quad d_{n} \in \mathbf{C}
$$

This implies that the zeros of $y_{1}(x)$ shrink to $x=0$, when $\nu \rightarrow 0$. Namely, for $j=1,2$ and $k \geq 0$, we have

$$
x_{k}(j) \rightarrow 0 \quad \text { when } \nu \rightarrow 0
$$

We prove that, for $x \neq 0$, the following holds:

$$
\lim _{\nu \rightarrow 0} y_{1}(x)=\left(\frac{1}{2}-\mu\right)\left[\left(\mu-\frac{1}{2}\right)\left(\ln (x)+d_{1}\right)-2\right]\left(\ln x+d_{1}\right)
$$

In the same way, $y_{n}(x)$ converges to a polynomial of $\ln x$, when $\nu \rightarrow 0$. We have proved this up to $n=4$ and conjecture that this is true for any $n$. Therefore, we expect that, when $x \neq 0$, the limit of $y(x)$ for $\nu \rightarrow 0$ exists, whith asymptotic expansion

$$
\lim _{\nu \rightarrow 0} y(x) \sim\left[\sum_{n=1}^{\infty} x^{n-1} P_{n}(\ln x)\right]^{-1}, \quad x \rightarrow 0
$$

where $P_{n}(\ln x)$ are certain polynomials of $\ln x$. On the other hand, we showed in [13] that, for $\nu=0, P V I_{\mu}$ has solutions with asymptotic expansion coinciding with the right hand side of the above (9). This verifies the conjecture. The Chazy solutions of [22] are also re obtained from the limit of solutions (1) for $\nu \rightarrow 0$.

In Section 4 the example of Picard solutions is didactically discussed, and we prove convergence of (5). In Section 5 we sketch the case of the general PVI equation. The rest of the paper is devoted to the proof of the theorems.

\section{Parameterization in terms of Monodromy Data}

The equation $P V I_{\mu}$ is associated to a semisimple Frobenius manifold of complex dimension 3, locally described by a system of canonical complex coordinates $u_{1}, u_{2}, u_{3}$. It is known that a branch of a solution is parameterized by the monodromy data of the manifold, namely $\mu$ and the three entries $s_{12}, s_{13}, s_{23}$ of the Stokes' matrix of the manifold, while the independent variable is $x=\left(u_{3}-u_{1}\right) /\left(u_{2}-u_{1}\right)$. These results are estabilished in [5]. A remarkable example is the Frobenius manifold given by the Quantum Cohomology of $C P^{2}[5]$. In this case,

$$
\mu=-1, \quad s_{12}=s_{13}=s_{23}=3
$$

(for the compution of the stokes matrix of $C P^{d}$ see [5] when $d=2$, and [8] when $d \geq 3$ ).

In place of $\mu$ and the entries of the Stokes matrix, we will use the equivalent quantities

$$
\theta_{\infty}:=2 \mu, \quad p_{0 x}:=2-s_{13}^{3}, \quad p_{01}:=2-s_{12}^{2}, \quad p_{x 1}:=2-s_{23}^{2}
$$

The above are usually employed in the isomonodromy preserving deformation approach to PVI, estabilished in [20] and [19]. They are monodromy data of the following $2 \times 2$ Fuchsian system of ODE associated to $P V I_{\mu}$ :

$$
\frac{d \Psi}{d \lambda}=\left[\frac{A_{0}(x)}{\lambda}+\frac{A_{x}(x)}{\lambda-x}+\frac{A_{1}(x)}{\lambda-1}\right] \Psi, \quad \lambda \in \mathbf{C} .
$$




$$
A_{0}+A_{1}+A_{x}=\operatorname{diag}\left(-\frac{\theta_{\infty}}{2}, \frac{\theta_{\infty}}{2}\right), \quad \text { Eigenvalues }\left(A_{i}\right)=0, \quad i=0,1, x ;
$$

A ordered base of loops $\Gamma$ is chosen in the fundamental group of $\mathbf{C} \backslash\{0, x, 1\}$. The three basic loops encircle $0, x$ and 1 respectively. The matrices $A_{i}(x)$ depend on $x$ in such a way that the monodromy group w.r.t. $\Gamma$ is independent of small deformations of $x$. By small deformation it is meant that $x$ does not go around a loop around 0 or 1 , namely that some banch cuts are chosen. For example, we may choose $-\pi \leq \arg x<\pi$ and $-\pi \leq \arg (x-1)<\pi$. Correspondingly, the solution $y(x)$ of $P V I_{\mu}$ is to be regarded as a branch. For the local analysis around $x=0$, we just consider $-\pi \leq \arg x<\pi$. Let $M_{0}, M_{x}, M_{1}$ be the monodromy matrices of a fundamental solution of the Fuchsian system w.r.t $\Gamma$. Let $M_{\infty}=\left(M_{1} M_{x} M_{0}\right)^{-1}$ be the monodromy at infinity. According to [20] and [19] the quantities $p_{i j}$ are:

$$
p_{i j}=\operatorname{Tr}\left(M_{i} M_{j}\right), \quad j=0, x, 1 .
$$

Let also

$$
p_{\infty}:=\operatorname{Tr} M_{\infty}=2 \cos \left(\pi \theta_{\infty}\right),
$$

The $p_{i j}$ 's and $p_{\infty}$ are coordinates for the space of monodromy data of the class of Fuchsian systems above. This space is an affine cubic surface [17] [19]:

$$
p_{0 x}^{2}+p_{01}^{2}+p_{x 1}^{2}+p_{0 x} p_{01} p_{x 1}-2\left(p_{0 x}+p_{01}+p_{x 1}\right)\left(2+p_{\infty}\right)+8+p_{\infty}^{2}+8 p_{\infty}=0
$$

Note that $\theta_{\infty}=2 \mu$ is fixed by the equation and $p_{0 x}, p_{01}, p_{x 1}$ are not independent, because of the cubic relation. Accordingly, only two complex parameters are free. The following facts follow from the general theory of Painlevé VI (see [14], section $2^{1}$ ):

Let the basis of loops $\Gamma$ be fixed.

1) If the monodromy group $<M_{0}, M_{x}, M_{1}>$ is not reducible, or $M_{\infty} \neq I$, the above $p_{\infty}$, $p_{i j}$ 's are a good system of coordinates for the monodromy group [17],[19].

2) If $M_{\infty} \neq I$, there is a one to one correspondence between a branch $y(x)$ and a point in the space of monodromy data.

3) If the monodromy group $<M_{0}, M_{x}, M_{1}>$ is not reducible and $M_{\infty} \neq I$, a branch $y(x)$ of a transcendent of $P V I_{\mu}$ is uniquely parameterized by the $p_{\infty}$ 's (i.e. $\theta_{\infty}$ ) and $p_{i j}$ 's, to which it is in one to one correspondence.

As a consequence of 3 ) above, the two complex integration constants of the branch $y(x)$ are functions of $p_{\infty}$ (i.e. $\theta_{\infty}$ ) and $p_{i j}$. A remarkable fact, established in Jimbo's paper [19], is that this parametrization is explicit, namely the integration constants are elementary or classical transcendental functions of $p_{\infty}, p_{i j}, i, j=0, x, 1$. Jimbo computed the parametrization for the generic PVI and most of the range of $p_{i j}$, except for $p_{i j}<-2{ }^{3}$

\footnotetext{
${ }^{1}$ Keep into account that, for $P V I_{\mu}$, each of the monodromy matrices $M_{0}, M_{x}$ and $M_{1}$ has a Jordan form $\left(\begin{array}{cc}1 & 2 \pi i \\ 0 & 1\end{array}\right)$ (but they cannot be put simultaneously in upper triangular form, in general), so they are not the identity matrix $I$,

${ }^{2}$ When the monodromy group is reducible, but $M_{\infty}$ is not the identity, the one to one correspondence between a point in the space of monodromy data and a branch still holds, but the $p_{\infty}, p_{i j}$ 's are no longer a good parametrization. The solutions in this case are known (see the Riccati solutions [27], [21]).

${ }^{3}$ As a consequence of this explicit parameterization of the three couples of integration constants at the three critical points in terms of the same monodromy data, the connection problem is solved. This is precisely the power of the method of monodromy preserving deformations.
} 
Solutions (1) occur when $s_{13}>2$, namely $p_{0 x}<-2$. In this case, the parametrization of $\nu$ and $d$ in terms of the monodromy data is computed in [14], and it is summarized in the following:

Proposition 1 Let $P V I_{\mu}$ be given, namely let $\mu$ be given. The branch (1) is associated to monodromy data such that $p_{0 x}<-2$. The real integration constant $\nu>0$ is obtained from

$$
p_{0 x}=-2 \cosh (2 \pi \nu) \quad\left(\text { namely, } s_{13}=2 \cosh (\pi \nu)\right)
$$

Therefore:

$$
\nu=\frac{2 \ln G}{\pi}, \quad G:=\left[\frac{\sqrt{p_{0 x}^{2}-4}-p_{0 x}}{2}\right]^{\frac{1}{4}}>1,
$$

$\diamond$ If $2 \mu \neq 2 i \nu+2 m+1, m \in \mathbf{Z}$, the complex integration constant $d$ is:

$$
\begin{aligned}
& d=\frac{i}{2} \ln \left\{-\frac{416^{2 i \nu} \Gamma\left(\frac{3}{2}-\mu-i \nu\right)^{2} \Gamma\left(\mu+\frac{1}{2}-i \nu\right)^{2}}{(2 \nu+}\right.i(1-2 \mu))^{2} \nu^{2} \sinh (2 \pi \nu)^{2} \Gamma(-i \nu)^{4} \\
&\left.\times\left[\frac{1}{2}\left(e^{2 \pi \nu} p_{x 1}-p_{01}\right) \sinh (2 \pi \nu)+(\cos (2 \pi \mu)+1)\left(e^{2 \pi \nu}+1\right)\right]\right\} .
\end{aligned}
$$

$\diamond$ If $2 \mu=2 i \nu+2 m+1, m \in \mathbf{Z}$, then $p_{0 x}=2 \cos (2 \pi \mu), p_{01}-2=\left(p_{x 1}-2\right) e^{ \pm 2 \pi \nu}$, and $d$ is as below:

$$
\begin{aligned}
& \text { - If } 2 \mu=2 i \nu+1-2 m, m=1,2,3, \ldots \text { : } \\
& d=-\frac{i}{2} \ln \left\{\frac{\nu^{2} \Gamma(m)^{2} \Gamma(2 i \nu+1-m)^{2}}{16^{2 i \nu} \Gamma(1+i \nu)^{4}}\left(p_{x 1}-2\right)\right\} \\
& 2-p_{01}=\left(2-p_{x 1}\right) e^{2 \pi \nu} \\
& \text { - If } 2 \mu=2 i \nu+1-2 m, m=-1,-2,-3, \ldots \text { : } \\
& d=\frac{i}{2} \ln \left\{\frac{\sinh (\pi \nu)^{4} 16^{2 i \nu} \Gamma(1-m)^{2} \Gamma(1+i \nu)^{4} \Gamma(m-2 i \nu)^{2}}{\nu^{2} \pi^{4}}\left(p_{x 1}-2\right)\right\} \\
& 2-p_{01}=\left(2-p_{x 1}\right) e^{-2 \pi \nu} \\
& \text { - If } 2 \mu=-2 i \nu+2 m-1, m=1,2,3, \ldots \text { (but } m \neq 1 \text { in (1)): } \\
& d=-\frac{i}{2} \ln \left\{\frac{\nu^{2} \Gamma(2 i \nu+1-m)^{2} \Gamma(m)^{2}}{16^{2 i \nu} \Gamma(1+i \nu)^{4}}\left(p_{x 1}-2\right)\right\} \\
& 2-p_{01}=\left(2-p_{x 1}\right) e^{2 \pi \nu} \\
& \text { - If } 2 \mu=-2 i \nu+2 m-1, m=0,-1,-2,-3, \ldots \text { : } \\
& d=\frac{i}{2} \ln \left\{\frac{\sinh (\pi \nu)^{4} 16^{2 i \nu} \Gamma(1-m)^{2} \Gamma(1+i \nu)^{4} \Gamma(m-2 i \nu)^{2}}{\nu^{2} \pi^{4}}\left(p_{x 1}-2\right)\right\} \\
& 2-p_{01}=\left(2-p_{x 1}\right) e^{-2 \pi \nu}
\end{aligned}
$$

Being $\nu \neq 0$, in the above cases $p_{01} \neq p_{x 1}$. 
Proof: Section 8.

Note that the freedom in the choice of the branch of the logarithm defines $d$ up to $d \mapsto d+k \pi$, $k \in \mathbf{Z}$. Such freedom does not affect $y(x)$ and we can choose $0 \leq \Re d \leq \pi$, as in (4).

For a given $P V I_{\mu}$, let $\nu>0$ and $d \in \mathbf{C}$ be given (equivalently, let monodromy data be given). Let us denote

$$
y(x, \nu, d):=y(x) \text { with behavior (1) with }-\pi \leq \arg x<\pi
$$

This is the branch with behavior (1). Its analytic continuation when $x$ goes around a small loop around $x=0$, or $x=1$, or $x=\infty$ may have a behavior different from (1). For the local analysis at $x=0$, it is enough to consider the analytic continuation when $x$ goes around a loop around $x=0$, namely $x \mapsto x e^{2 \pi i}(|x|<1)$. The new branch is parametrized in terms of new monodromy data $p_{i j}^{\prime}, i, j \in\{0, x, 1\}$, computable by an action of the braid group as follows (see $[6])$ :

$$
\begin{gathered}
p_{0 x}^{\prime}=p_{0 x}, \quad p_{01}^{\prime}=-p_{01}+2 p_{x 1} \cosh (2 \pi \nu)+4 \cos (2 \pi \mu)+4 \\
p_{x 1}^{\prime}=p_{x 1}\left(4 \cosh (2 \pi \nu)^{2}-1\right)-2 \cosh (2 \pi \nu) p_{01}+4(\cos (2 \pi \mu)+1)(2 \cosh (2 \pi \nu)+1)
\end{gathered}
$$

We see that $p_{0 x}^{\prime}=p_{0 x}<-2$, thus the new branch has again a behavior (1) and Proposition 1 holds. In particular, $\nu$ is unchanged. As a consequence, we have:

Proposition 2 The analytic continuation of the branch $y(x, \nu, d)$ corresponding to the loop $x \mapsto x e^{2 \pi i}$ is:

$$
y(x, \nu, d) \mapsto y(x, \nu, d+2 \pi i \nu)=y\left(x e^{2 \pi i}, \nu, d\right), \quad-\pi \leq \arg x<\pi .
$$

Proof: Substituting into the formulas of Proposition 1 the expressions of $p_{x 1}^{\prime}$ and $p_{01}^{\prime}$ and simplifying, we find that the new $d$, denoted $d^{\prime}$, is $d^{\prime}=d+2 \pi i \nu$.. Observe also that that $\exp \left\{2 i d^{\prime}\right\} x^{2 i \nu}=\exp \{2 i d\}\left(x e^{2 \pi i}\right)^{2 i \nu}$. Immediately (18) follows.

Remark on the poles of a branch: The rays of Theorem 1, where the zeros $x_{k}(1)$ and $x_{k}(2)$ lie, may be outside the range $-\pi \leq \arg x<\pi$, depending on their angles $\frac{\Im d}{\nu}$ and $\frac{\Im d}{\nu}+$ $\frac{1}{\nu} \ln \left|\frac{2 \mu-1+2 i \nu}{2 \mu-1-2 i \nu}\right|$ (note: the expansion (1) is defined for $|\arg x|<\vartheta$, with $\vartheta>0$ and $|x| \neq 0$ sufficently small. Recall that $\vartheta$ is arbitrary (but fixed) and this fact allows to find zeros with angles outside $-\pi \leq \arg x<\pi$ ). When this happens, one or both the sequences of the zeros do not fall in the domain $-\pi \leq \arg x<\pi$. Accordingly, the branch $y(x, \nu, d)$ does not have poles. The analytic continuation of the branch when $x$ goes around a loop $x \mapsto x e^{2 \pi i}(|x|<1)$ is (18). The shift $d \mapsto d+2 \pi i \nu$ changes the immaginary exponent of the $x_{k}(1)$ 's by $-2 \pi i$. This implies that, by a sufficient number of loops, we can always find a branch with poles, namely such that at least one of the two sequences of zeros is in the range $-\pi \leq \arg x<\pi$.

\section{Limit for $\nu \rightarrow 0$}

Suppose that $d$ vanishes with $\nu$ as $\nu \rightarrow 0$. Namely:

$$
d=d_{1} \nu\left(1+d_{2}(\nu)\right), \quad d_{1} \in \mathbf{C} \backslash\{0\}, \quad d_{2}(\nu) \rightarrow 0, \quad \nu \rightarrow 0 .
$$

If this happens, the zeros $x_{k}(j)$ of Theorem 1 shrink to $x=0$, provided that $k \geq 0 .{ }^{4}$

\footnotetext{
${ }^{4}$ As for $x_{k}(2)$, use the fact that: $\exp \left\{-\frac{i}{2} \ln \left(\frac{2 \mu-1+2 i \nu}{2 \mu-1-2 i \nu}\right)\right\}=\exp \left\{-\frac{i}{2} \ln \left(1+\frac{4 \nu i}{2 \mu-1}+O\left(\nu^{2}\right)\right)\right\}=\frac{4}{2 \mu-1}+O(\nu), \quad \nu \rightarrow 0$.
} 
Proposition 3 Suppose that $d$ is as in (19). Then, for $x \neq 0$, there exists:

$$
\lim _{\nu \rightarrow 0} y_{1}(x)=P_{1}(\ln x)
$$

where $P_{1}(\ln x)$ is the polynomial of $\ln x$ appearing in the r.h.s. of (8).

Proof: Substitute $d=d_{1} \nu\left(1+d_{2}(\nu)\right)$ in (3). Then expand $\exp \{2 i d\}=1+2 i d_{1} \nu+o(\nu)$ and $x^{2 i \nu}=1+2 i \nu \ln x+O\left(\nu^{2}\right)$. The structure of the coefficients $A_{1,-1}, A_{10}, A_{11}$ allows simplification of the divergences $\nu^{-2}$ and $\nu^{-1}$ contained in the coefficients themselves. Therefore, $y_{1}(x)$ is expanded in series for $\nu \rightarrow 0$, which by direct computation is easily verified to be:

$$
y_{1}(x)=P_{1}(\ln x)+\nu(\text { polynomial } \ln x)+\nu^{2}(\text { polynomial } \ln x)+\ldots, \quad x \neq 0
$$

Remark: If $d=d_{0}+d_{1} \nu+d_{2} \nu^{2}+\ldots, d_{0} \neq 0$, then

$y_{1}(x)=\frac{(2 \mu-1)^{2}\left(\cos \left(2 d_{0}\right)-1\right)}{8 \nu^{2}}-\frac{(2 \mu-1)\left((2 \mu-1)\left(\ln x+d_{1}\right)-2\right) \sin \left(2 d_{0}\right)}{4 \nu}+O\left(\nu^{0}\right), \quad \nu \rightarrow 0$

Thus, the limit for $\nu \rightarrow 0$ and $x \neq 0$ does not exist.

The same computation of the proof of Proposition 3 can be done for $y_{n}(x)$. We verified, up to $n=4$, that the divergences in $A_{n m}$ for $\nu \rightarrow 0$ are canceled by the expansion of $\exp \left\{2 i\left(d_{1} \nu(1+\right.\right.$ $\left.\left.d_{2}(\nu)\right)\right\}$ and $x^{2 i \nu}=\exp \{2 i \nu \ln x\}$ for $\nu \rightarrow 0$, so that there exists:

$$
\lim _{\nu \rightarrow 0} y_{n}(x)=P_{n}(\ln x)
$$

where:

$$
P_{n}(\ln x)=(2 \mu-1) p_{0}\left(\mu, d_{1}\right)+\sum_{N=1}^{2 n}(2 \mu-1)^{N} p_{N}\left(\mu, d_{1}\right) \ln ^{N}(x)
$$

The $p_{N}\left(\mu, d_{1}\right)$ are certain polynomials of $\mu$ and $d_{1}$. For example:

$$
\begin{gathered}
P_{2}(\ln x)=-\frac{(2 \mu-1)^{4}}{32} \ln ^{4}(x)-\frac{(2 \mu-1)^{3}\left((2 \mu-1) d_{1}-2\right)}{8} \ln ^{3}(x)+ \\
-3\left(\mu-\frac{1}{2}\right)^{2}\left(\frac{5}{6}+\left(\mu-\frac{1}{2}\right)^{2} d_{1}^{2}+(1-2 \mu) d_{1}\right) \ln ^{2}(x)+ \\
-(2 \mu-1)\left(\left(\mu-\frac{1}{2}\right)^{3} d_{1}^{3}-3\left(\mu-\frac{1}{2}\right)^{2} d_{1}^{2}+\frac{5}{4}\left((2 \mu-1) d_{1}+\frac{1}{2} \mu-\frac{3}{4}\right) \ln (x)+\right. \\
-(2 \mu-1)\left(2+\left(\mu-\frac{1}{2}\right)^{2} d_{1}^{3}+\frac{3}{2}(1-2 \mu) d_{1}^{2}+2 d_{1}\right)\left(\left(\mu-\frac{1}{2}\right) d_{1}-1\right)
\end{gathered}
$$

Vanishing of $d$ as $\nu \rightarrow 0$ is not an arbitrary assumption, because the following holds:

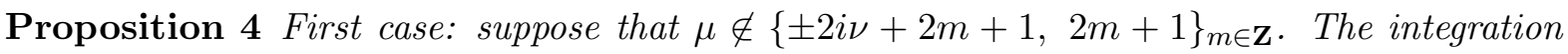
constant $d$, given by (13), is expanded as a Taylor series, convergent for $\nu$ sufficiently small:

$$
d=\sum_{n=1}^{\infty} d_{n} \nu^{n}, \quad \nu \rightarrow 0, \quad d_{n} \in \mathbf{C}
$$


where

$$
d_{1}=2 \gamma-4 \ln 2+\frac{i \pi}{2}+2 \psi\left(\mu+\frac{1}{2}\right)-\pi \tan (\pi \mu)+\frac{i \pi\left(p_{x 1}-p_{01}\right)}{8 \cos ^{2}(\pi \mu)}
$$

Second case: when $\mu= \pm 2 i \nu+2 m+1, m \in \mathbf{Z}$. The integration constant $d$ is expanded as a Taylor series, convergent for $\nu$ sufficiently small, as follows:

- The cases of (14) and (16):

$$
\begin{gathered}
d=-\frac{i}{2} \ln \left(\frac{2-p_{x 1}}{4}\right)+2(\psi(m)+\gamma-2 \ln 2) \nu+\sum_{n=2}^{\infty} d_{n} \nu^{n}, \\
m=1,2,3, \ldots, \quad d_{n} \in \mathbf{C}
\end{gathered}
$$

- The case of (15) and (17):

$$
\begin{gathered}
d=\frac{i}{2} \ln \left(\frac{2-p_{x 1}}{4}\right)+2(\psi(1-m)+\gamma-2 \ln 2) \nu+\sum_{n=2}^{\infty} d_{n} \nu^{n}, \\
m=0,-1,-2,-3, \ldots, \quad d_{n} \in \mathbf{C}
\end{gathered}
$$

In the above formulas $\psi(m)+\gamma=\sum_{k=1}^{m-1} \frac{1}{k}$.

Proof: Section 10.

The above results allow us to formulate the following:

Conjecture: Let $\mu \neq \frac{1}{2}$ and assume that $d$ is as in (19). Let $y(x)$ be the branch of (1). Then, for $x \neq 0$, there exist the limit of $y(x)$ for $\nu \rightarrow 0$, with asymptotic series (9).

Recall that $p_{0 x}=-2 \cosh (2 \pi \nu)$. If $\nu \rightarrow 0$, then $p_{0 x} \rightarrow-2$. The result established in [13] (see also [14] for a review) states that to the monodromy data such $p_{0 x}=-2$ and $p_{x 1}, p_{01}$ arbitrary, there is associated a branch of a solution of $P V I_{\mu}, \mu \neq \frac{1}{2}$, with critical behavior:

$$
y(x)=\frac{1}{P_{1}(\ln x)}\left(1+O\left(x \ln ^{2}(x)\right)\right)=-\frac{4}{(2 \mu-1)^{2} \ln ^{2}(x)}\left(1+O\left(\frac{1}{\ln x}\right)\right), \quad x \rightarrow 0
$$

The full asymptotic expansion can be computed (by substitution into the equation) and it coincides with (9). This shows that the conjecture is true. Note that the monodromy data, to which a branch is in one to one correspondence, are contained in $d_{1}$, when $\mu \neq \pm 2 i \nu+2 m+1$. Also note that the first term in the critical behavior (20) does not depend on $d_{1}$, namely on the monodromy data $p_{x 1}, p_{01}$.

\subsection{Chazy Solutions, $\nu \rightarrow 0$ and $\mu \rightarrow-\frac{1}{2}$}

In the special cases $\mu= \pm 2 i \nu+2 m+1, d$ does not vanish, therefore the limit of $y_{1}(x)$ for $\nu \rightarrow 0$ and $x \neq 0$ diverges as $\nu^{-2}$, except possibly when $p_{x 1}=-2$. Also note that the restriction $\mu \neq \frac{1}{2}+m, m \in \mathbf{Z}$ avoids divergences of $d$ in Proposition 4 . Such cases may be analyzed separately. Preliminarily, we observe that the symmetries of PVI imply that one can always assume that $-1 \leq \Re \mu<0$. Thus, it is enough to analyze the case $\mu=-\frac{1}{2}$. We will limit ourselves to the analysis of the solutions associated to monodromy data which satisfy the condition:

$$
p_{x 1}=p_{01}=p_{0 x}=-2 \cosh (2 \pi \nu)
$$




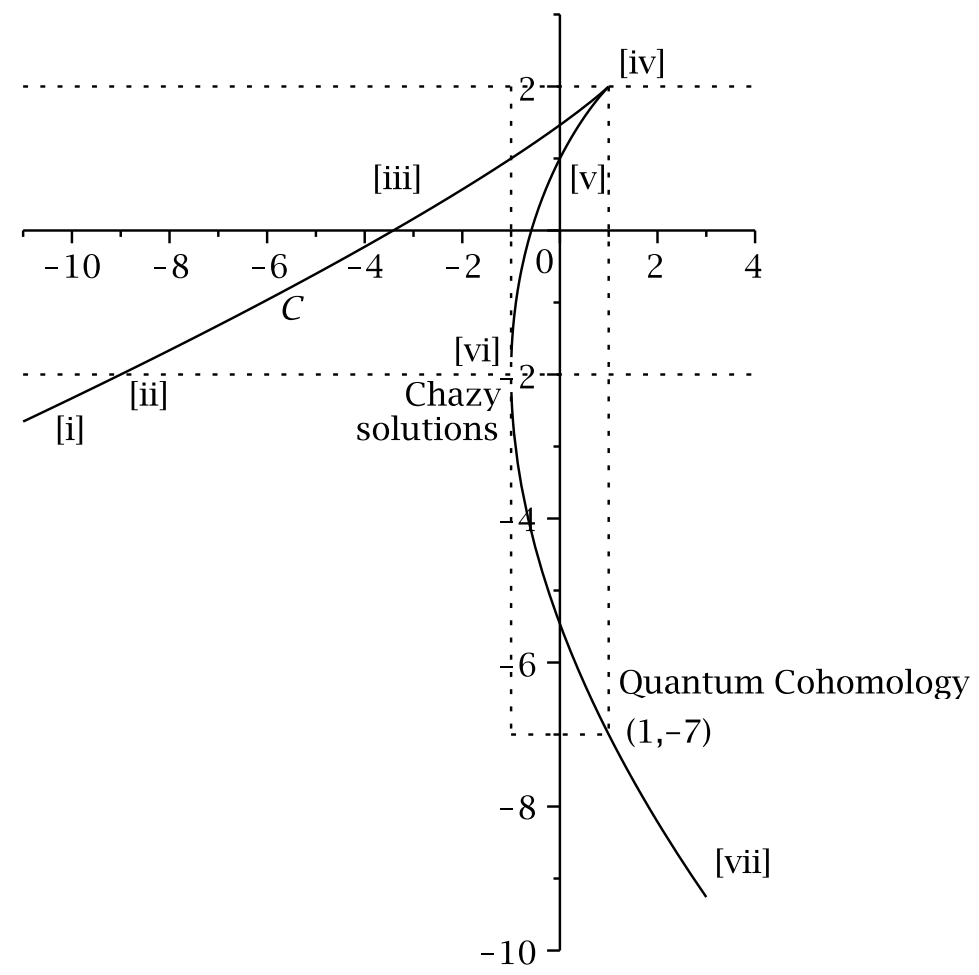

Figure 4: Cubic curve in the plane $\left(\cos \pi \theta_{\infty}, p_{0 x}\right)=\left(\cos (2 \pi \mu), p_{0 x}\right)$

The cubic relation (11) becomes:

$$
3 p_{0 x}^{2}+p_{0 x}^{3}-12 p_{0 x}(1+\cos (2 \pi \mu))+4 \cos ^{2}(2 \pi \mu)+16 \cos (2 \pi \mu)+8=0
$$

This curve is represented in figure 4 by the continuous line, when $\cos (2 \pi \mu)$ is real. Observe that $\nu \geq 0$ is well defined when $p_{0 x} \leq-2$, This condition singles out two portions of the curve, namely $[\mathrm{i}] \cup[\mathrm{ii}]$ and $[\mathrm{vi}] \cup[$ vii]. If we require that also $\mu$ is real, namely $-1 \leq \cos (2 \pi \mu) \leq 1$, the condition singles out only the portion of the curve between $(-1,-2)$ and $(1,-7)$. In this case, the functional relation between $\mu$ and $\nu$ is established in Lemma 2 of Section 11:

$$
\begin{gathered}
\mu=-\frac{1}{2}+\frac{1}{2 \pi} \arg \left(c_{1}(\nu)-\frac{i}{2} \sqrt{c_{2}(\nu)}\right), \\
\left\{\begin{array}{cc}
c_{1}(\nu):= & 2+3 \cosh (2 \pi \nu)-\cosh (3 \pi \nu)-3 \cosh (\pi \nu), \\
c_{2}(\nu):= & 96 \cosh (\pi \nu)+52 \cosh (3 \pi \nu)+12 \cosh (5 \pi \nu)+ \\
& -50-78 \cosh (2 \pi \nu)-30 \cosh (4 \pi \nu)-2 \cosh (6 \pi \nu)
\end{array}\right.
\end{gathered}
$$

where $0 \leq \nu \leq 2 \ln \mathbf{G} / \pi$ and $\mathbf{G}=(1+\sqrt{5}) / 2$. The discriminat $c_{2}(\nu) \geq 0$ and the square root is the positive one. When we choose the argument with determination

$$
-\pi \leq \arg \left(c_{1}(\nu)-\frac{i}{2} \sqrt{c_{2}(\nu)}\right) \leq 0
$$

it follows that $\mu$ can be expanded as a convergent series, as in the following proposition. 
Proposition 5 Let $p_{x 1}=p_{01}=p_{0 x}=-2 \cosh (2 \pi \nu) \leq-2$ and let $\mu$ be real, $-1 \leq \mu<0$. In this case, $\mu$ and the integration constant $d$ have a convergent Taylor series:

$$
\left\{\begin{array}{l}
\mu=\sum_{n=0}^{\infty} \mu_{n} \nu^{2 n} \\
d=\sum_{n=0}^{\infty} d_{2 n+1} \nu^{2 n+1}
\end{array}, \quad \nu \rightarrow 0\right.
$$

The first terms are

$$
\mu_{0}=-\frac{1}{2}, \quad \mu_{2}=-\frac{\pi \sqrt{3}}{2}, \quad \mu_{4}=-\frac{\sqrt{3} \pi^{3}}{8}, \quad \mu_{6}=-\frac{17}{240} \pi^{5} \sqrt{3}
$$

and

$$
\begin{gathered}
d_{1}=\frac{i \pi}{2}-4 \ln (2)-\frac{\pi \sqrt{3}}{2}, \quad d_{3}=0, \quad d_{5}=-\left[\frac{3 \zeta(3)}{2}+\frac{\pi^{3} \sqrt{3}}{30}\right] \pi^{2}, \\
d_{7}=\left[3 \zeta(5)-\frac{3 \pi^{2} \zeta(3)}{4}-\frac{83 \pi^{5} \sqrt{3}}{7560}\right] \pi^{2} .
\end{gathered}
$$

Proof: Section 11.

The analogous of Proposition 3 holds:

Proposition 6 Consider the branch (1) associated to the monodromy data $\mu \in \mathbf{R}$ and $p_{1 x}=$ $p_{01}=p_{0 x}=-2 \cosh (2 \pi \nu)$. If $\nu \rightarrow 0$, then $\mu \rightarrow-\frac{1}{2}$ and, for $x \neq 0$, there exists:

$$
\lim _{\nu \rightarrow 0} y_{1}(x)=P_{1}^{(-1 / 2)}(\ln x)
$$

where $P_{1}^{(-1 / 2)}(\ln x)=-\left(\ln x+d_{1}+2\right)\left(\ln x+d_{1}\right)$ and $d_{1}=\frac{i \pi}{2}-4 \ln (2)-\frac{\pi \sqrt{3}}{2}$.

Proof: $\mu \rightarrow-\frac{1}{2}$ because of Proposition 5. Substitute into (3) the series of $d$ and $\mu$ of Proposition 5 and expand $\exp \{2 i d\}=1+2 i d_{1} \nu+o(\nu)$ and $x^{2 i \nu}=1+2 i \nu \ln x+O\left(\nu^{2}\right)$. The structure of the coefficients $A_{1,-1}, A_{10}, A_{11}$ allows simplification of the divergences $\nu^{-2}$ and $\nu^{-1}$ contained in the coefficients themselves. Therefore, $y_{1}(x)$ is expanded in series for $\nu \rightarrow 0$, and by direct computation it is easily verified that $y_{1}(x) \rightarrow P_{1}^{(-1 / 2)}(\ln x)$ when $\nu \rightarrow 0$.

We verified, up to $n=4$, that every $y_{n}(x)$ converges, for $\nu \rightarrow 0$, to a polynomial $P_{n}^{(-1 / 2)}(\ln x)$. We conjecture again that if $\mu=-\frac{1}{2}$, to the monodromy data $p_{0 x}=p_{x 1}=p_{01}=-2$ a branch $y(x)$ is associated with asymptotic behavior

$$
\frac{1}{y(x)} \sim \sum_{n=1}^{\infty} x^{n-1} P_{n}^{(-1 / 2)}(\ln x), \quad x \rightarrow 0
$$

where

$$
P_{n}^{(-1 / 2)}(\ln x)=\sum_{N=0}^{2 n} p_{N} \ln ^{N}(x), \quad p_{N} \in \mathbf{C}
$$

The conjecture is true. It is well known that when $\mu=-\frac{1}{2}$, to the monodromy data $p_{0 x}=$ $p_{x 1}=p_{01}=-2$ a one parameter class of Chazy solutions of $P V I_{-\frac{1}{2}}$ is associated. The result is established in [22]. Such solutions form a one parameter class, which includes (23) (no parameter in (23)). Therefore, the limit of (1) for $\nu \rightarrow 0, p_{0 x}=p_{x 1}=p_{01}$ and $\mu$ real, is one element in the class of Chazy solutions. Similar result is estabilished in Section 3.1, Lemma 9, of $[22]$. 


\section{Example of Picard Solutions}

Picard solutions [24] occurr for $\mu=\frac{1}{2}$. Their example shows that, when we consider $y(x)$ on the universal covering of the puntured neighborhood of zero, then the poles accumulate at zero along spirals. The Picard solutions of $P V I_{1 / 2}$ are

$$
y(x)=\wp\left(\nu_{1} \omega_{1}(x)+\nu_{2} \omega_{2}(x) ; \omega_{1}, \omega_{2}\right)+\frac{1+x}{3}, \quad \nu_{1}, \nu_{2} \in \mathbf{C},
$$

where the half-periods are $\omega_{1}(x)=\mathbf{K}(x)$ and $\omega_{2}(x)=i \mathbf{K}(1-x)$, and

$$
\mathbf{K}(x):=\int_{0}^{1} \frac{d \zeta}{\sqrt{\left(1-\zeta^{2}\right)\left(1-x \zeta^{2}\right)}}=\frac{\pi}{2} F\left(\frac{1}{2}, \frac{1}{2}, 1 ; x\right)
$$

A branch is fixed by the cuts $|\arg x|<\pi,|\arg (1-x)|<\pi$. For $|x|<1$ and $|\arg x|<\pi$, we can write

$$
\omega_{2}(x)=-\frac{i}{2}\left[F\left(\frac{1}{2}, \frac{1}{2}, 1 ; x\right) \ln (x)+F_{1}(x)\right], \quad|\arg x|<\pi
$$

where

$$
F_{1}(x)=\sum_{n=0}^{\infty} \frac{\left[\left(\frac{1}{2}\right)_{n}\right]^{2}}{(n !)^{2}} 2\left[\psi\left(n+\frac{1}{2}\right)-\psi(n+1)\right] x^{n}, \quad|x|<1
$$

Recal that $\psi(z)=\frac{d}{d z} \ln \Gamma(z), \psi\left(\frac{1}{2}\right)=-\gamma-2 \ln 2, \psi(1)=-\gamma$, and $\psi(a+n)=\psi(a)+\sum_{l=0}^{n-1} \frac{1}{a+l}$. The behavior of $y(x)$ at $x=0$ follows from the Fourier expansion of $\wp$ at $x=0$. When $x \rightarrow 0$ and $\nu_{2}=2 i \nu, \nu \in \mathbf{R}$, this is of type (1), with $d=\frac{\pi \nu_{1}}{2}-\nu \ln 16$.

$F_{1}(x)$ and $F(x)$ are single valued for $|x|<1$, and multi-valuedness of $\omega_{2}(x)$ comes form $\ln x$. Thus, $y(x)$ may be regarded as defined on the universal covering of $\mathbf{C} \backslash\{0,1, \infty\}$, and in particular, for $|x|<1$, on the universal covering of a punctured neighborhood of $x=0$. The poles on the universal covering of a punctured neighborhood of $x=0$ can be determined. They are a double sequence of points $\xi_{k N}$, solutions of the equation $\nu_{1} \omega_{1}(x)+\nu_{2} \omega_{2}(x)=$ $2 k \omega_{1}(x)-2 N \omega_{2}(x), k, N \in \mathbf{Z}$, namely:

$$
\frac{\nu_{2}+2 N}{2 i} \ln \frac{x}{16}+\frac{\pi \nu_{1}}{2}+\frac{\nu_{2}+2 N}{2 i}\left[\frac{F_{1}(x)}{F(x)}+\ln 16\right]=k \pi, \quad|x|<1,
$$

where $\frac{F_{1}}{F}+\ln 16$ vanishing as $x$, when $x \rightarrow 0$. Thus, one can write a pole as

$$
\xi_{k N}=x_{k N}\left(1+\delta\left(x_{k N}\right)\right)
$$

where $x_{k N}$ solves

$$
\frac{\nu_{2}+2 N}{2 i} \ln \left(\frac{x_{k N}}{16}\right)+\frac{\pi \nu_{1}}{2}=k \pi, \quad k \in \mathbf{Z} \quad \Longrightarrow \quad x_{k N}=16 \exp \left\{i \pi \frac{2 k-\nu_{1}}{2 N+\nu_{2}}\right\}
$$

The correction $\delta\left(x_{k N}\right)$ is expected to vanish if $x_{k N} \rightarrow 0$. It is solution of the equation

$$
\ln \left(1+\delta\left(x_{k N}\right)\right)+\frac{F_{1}\left(x_{k N}\left(1+\delta\left(x_{k N}\right)\right)\right)}{F\left(x_{k N}\left(1+\delta\left(x_{k N}\right)\right)\right)}+\ln 16=0
$$

One needs to notice the following facts: 


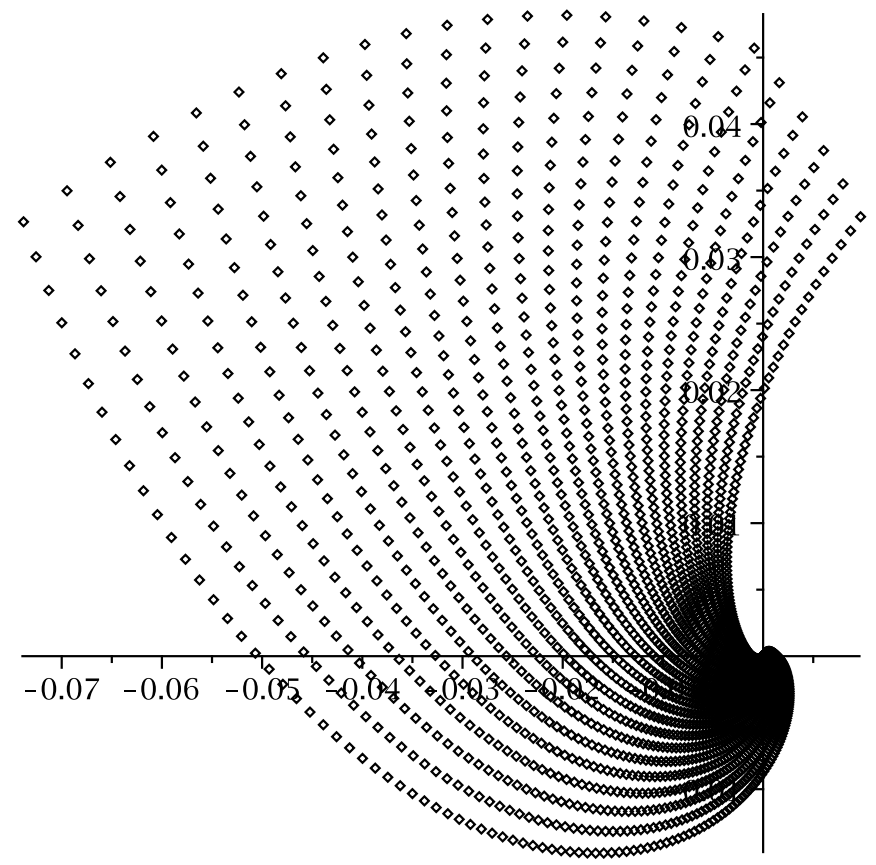

Figure 5: First approximation $x_{k N}$ of the poles of a Picard Solution on the universal covering, projected onto the $x$ plane. The figures shows the case $\nu_{1}=100-80 i, \nu_{2}=1-129 i$. Twenty four spirals are displayed, for $33 \leq N \leq 56$. For any $N$, the poles are along a spiral, accumulating at $x=0$ as $k$ increases. In the picture, $k>190$.

1) The $x_{k N}$ 's can be written as:

$$
\begin{gathered}
x_{k N}=x_{0 N} \exp \left\{2 \pi \frac{\Im \nu_{2}}{\left|\nu_{2}+2 N\right|^{2}} k\right\} \exp \left\{2 \pi i \frac{\Re \nu_{2}+2 N}{\left|\nu_{2}+2 N\right|^{2}} k\right\}, \\
\text { where } x_{0 N}=16 \exp \left\{-\frac{i \pi \nu_{1}}{\nu_{2}+2 N}\right\}
\end{gathered}
$$

In order to ensure that $\left|x_{k N}\right|<1$, the sign of $k$ is chosen $\operatorname{sgn}(k)=-\operatorname{sgn}\left(\Im \nu_{2}\right)$ and, for given $N,|k|$ is sufficiently big. The above form (26) makes it clear that for any fixed $N$, the $x_{k N}$ lie along a spiral, accumulating at $x=0$ as $k \rightarrow \infty$. The index $N$ singles out the spiral, while $k$ gives the dynamics of the $x_{k N}$ 's along that spiral. See figure 5 . On the other hand, if $k$ is fixed and $N$ varies, the typical distribution of the $x_{k N}$ 's is in figure 6 . For fixed $k, N$ cannot be too big, otherwise $\left|x_{k N}\right|$ becomes greater then 1 (and tends to 16 as $N \rightarrow \pm \infty$ ), in which case the local analysis makes no sense.

2) If $\Re \nu_{2}=0$, no $N$-spiral is a ray. If $\Re \nu_{2}=2 l, l \in \mathbf{Z}$, the spiral for $N=-l$ is a ray. For example, let $\nu_{2}=2 i \nu, \nu \in \mathbf{R}$. Then, the $x_{k 0}$ 's are $x_{k 0}=16 \exp \left\{-\frac{\pi \nu_{1}}{2 \nu}-\frac{k \pi}{\nu}\right\}$. They lie along the ray of angle $-\frac{\pi \Im \nu_{1}}{2 \nu}$, in a disk around $x=0$ of radius less than 1 , provided that $k>\frac{\nu}{\pi} \ln 16-\frac{1}{2} \Re \nu_{1}$. They are an example of the zeros of Theorem 1 .

3) Fix the branch cut $-\pi \leq \arg x<\pi$. Every spiral leaves the cut neighborhood of $x=0$ as $|k|$ increases, and this eventually happens for any $N$. Thus, there are no $x_{k N}$ in a sufficiently small neighborhood (with branch cut) of $x=0$, except possibly in the case $\Re \nu_{2}=2 l, l \in \mathbf{Z}$, when the $x_{k,-l}$ 's lie along a ray. 


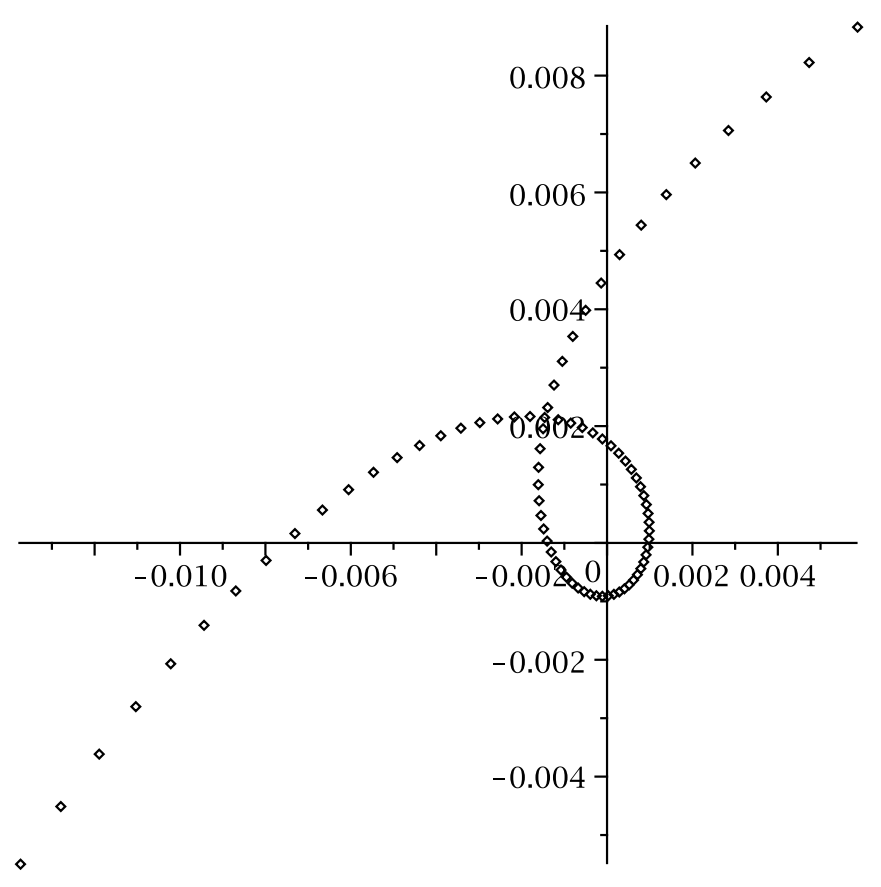

Figure 6: Case of figure $5\left(\nu_{1}=100-80 i, \nu_{2}=1-129 i\right)$, with fixed $k=250$ and varying $N$ $(-30 \leq N \leq 50)$.

Example: We consider the case $\nu_{1}=\nu_{2}=i / 3$. First, we find the zeros $x_{k N}$ which lie in a neighborhood of $x=0$, with radius less the 1 and branch cut $-\pi \leq \arg x<\pi$. The values of $N$ and $k$ are determined imposing $\left|x_{k N}\right|<1,-\pi \leq \arg x_{k N}<\pi$. This gives a system of inequalities

or

$$
\left\{\begin{array}{c}
k<-\frac{1}{3 \pi}\left(72 \ln (2) N^{2}+2 \ln (2)+3 \pi N\right) \\
k \leq-N, \quad \frac{18 N^{2}+1}{18 N}<k
\end{array} \quad \text { if } N<0\right.
$$

or

$$
\left\{\begin{array}{c}
k<-\frac{1}{3 \pi}\left(72 \ln (2) N^{2}+2 \ln (2)+3 \pi N\right) \\
k<\frac{18 N^{2}+1}{18 N}, \quad-N \leq k
\end{array} \quad \text { if } N>0\right.
$$

$$
k<-\frac{2 \ln (2)}{3 \pi}=-0.147 \ldots \quad \text { if } N=0
$$

The above are satisfied only for $N=0$ and $k \leq-1$, which means that only the ray occurring for $N=0$ (the negative real axis) is allowed. Then, we consider a portion of the universal covering, by imposing that $\left|x_{k N}\right|<1$ and $-25 \pi<\arg x_{k N}<23 \pi$. Again, one obtains inequalities, graphically represented in figure 7 . The points $(N, k)$ satisfying the inequalities are inside the region bounded by the curves of figure 7 . Therefore, the points $x_{k N}$ which lie in $\{x|| x \mid<1,-$ $25 \pi \leq \arg x<23 \pi\}$ are: the infinite sequence of poles on the negative real axis, corresponding to $N=0$ and $k \leq-1$, plus only a finite number of poles, corresponding to $-4 \leq N \leq 4, N \neq 0$, and $k$ inside the region bounded by the curves of figure 7 . They are represented in figures 8 and 9 . 


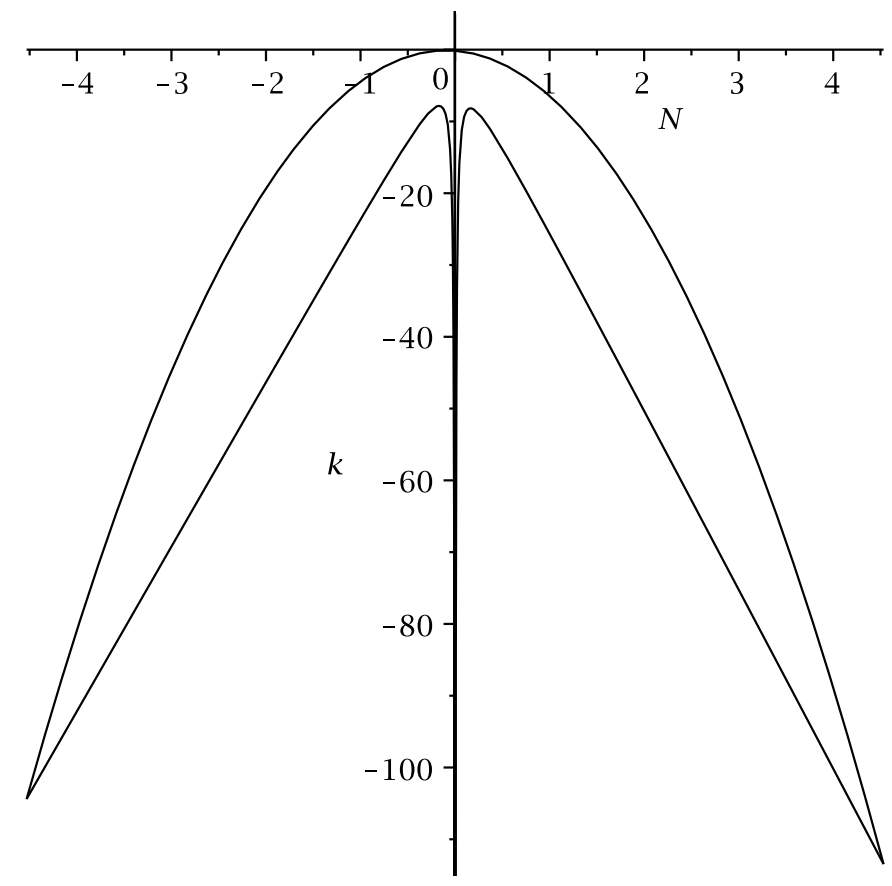

Figure 7: $\nu_{1}=\nu_{2}=i / 3$. $(N, k)$ plane. When $N$ and $k$ take values in the region delimited by the curves, the points $x_{k N}$ lie in $|x|<1$ with $-25 \pi \leq \arg x<23 \pi$. Allowed values of $N$ are $-4 \leq N \leq 4$. For $N=0$, any $k \leq-1$ is contained in the region $(N=0$ is a vertical asymptotic line for the curves).

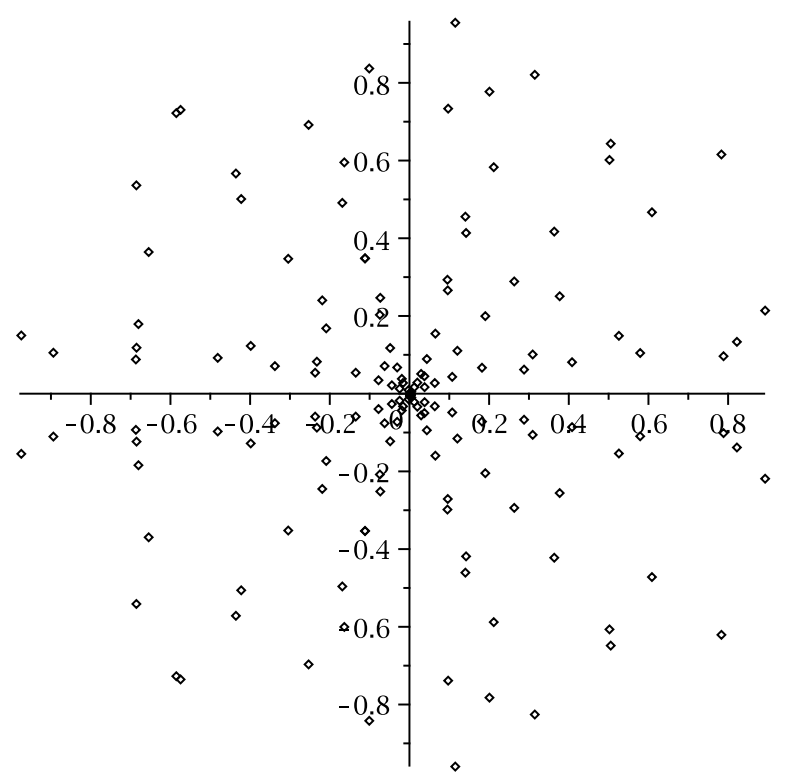

Figure 8: Figure shows the projection on the $x$-plane of the finite number of $x_{k N}$ 's, $N \neq 0$, which lie in $|x|<1$ with $-25 \pi<\arg x<23 \pi$, for $\nu_{1}=\nu_{2}=i / 3$. They exist for $-4 \leq N \leq 4$, $N \neq 0$ (for $N=0$ there is an infinite sequence $x_{k 0}$ on the negative real axis, accumulating at $x=0$, not depicted here). 


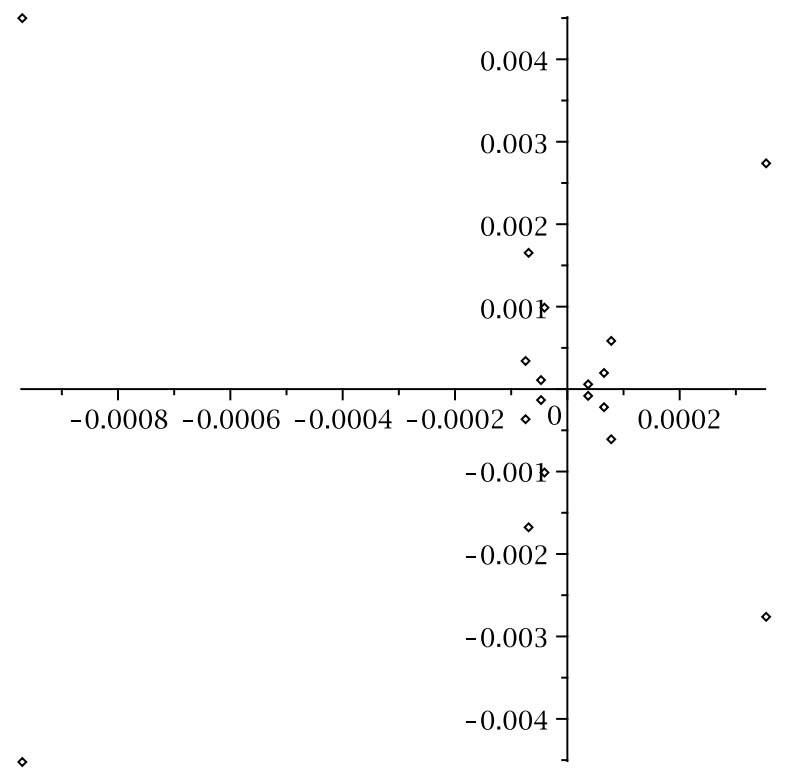

Figure 9: Zoom of figure 8. At the scale in the figure, only $N=-1$ and $N=1$ appear. $N=0$ is not represented. For $N \neq 0$, there are no $x_{k N}$ in a neighborhood sufficiently small of $x=0$.

We now analyze equation (25). It can be rewritten as

$$
x_{k N}\left(1+\delta\left(x_{k N}\right)\right)=x_{k N} \frac{1}{16} \exp \left\{-\frac{F_{1}\left(x_{k N}\left(1+\delta\left(x_{k N}\right)\right)\right)}{F\left(x_{k N}\left(1+\delta\left(x_{k N}\right)\right)\right)}\right\}
$$

Droping the indeces $k$ and $N$ and letting $\xi=x(1+\delta(x))$, the above is the equation

$$
\xi=x \frac{1}{16} \exp \left\{-\frac{F_{1}(\xi)}{F(\xi)}\right\}
$$

Lagrange inversion theorem can be applied to (28), because $\frac{1}{16} \exp \left\{\frac{-F_{1}(\xi)}{F(\xi)}\right\}$ is analytic inside a disk of radius $\xi_{0}<1$, centered at $\xi=0$. The condition on $x$ in order to apply the theorem is $|x|<|16 \xi|\left|\exp \left\{\frac{F_{1}(\xi)}{F(\xi)}\right\}\right|$, when $\xi$ is on the contour of the disk. For such $x$, Lagrange theorem ${ }^{5}$ says that (28) has a root with the following convergent series:

$$
\xi(x)=\left.\sum_{n=1}^{\infty} \frac{x^{n}}{n !} \frac{d^{n-1}}{d a^{n-1}}\left[\frac{1}{16^{n}} \exp \left\{-\frac{n F_{1}(a)}{F(a)}\right\}\right]\right|_{a=0}
$$

\footnotetext{
${ }^{5}$ Lagrange Inversion Theorem (Lagrange 1770 - see Whittaker \& Watson, a Course of Modern Analysis, pag 133): Let $\phi(z)$ be analytic on and inside a contour $C$ surrounding a point $a$, and let $x$ be such that

$$
|x \phi(z)|<|z-a|
$$

at all points $z \in C$. Then, the equation

$$
x \phi(\xi)=\xi-a
$$

has one root in the interior of $C$ :$$
\xi(x)=a+\sum_{n=1}^{\infty} \frac{x^{n}}{n !} \frac{d^{n-1}}{d a^{n-1}}\left[\phi(a)^{n}\right] .
$$ 


$$
=x-\frac{1}{2} x^{2}+\frac{11}{64} x^{3}-\frac{3}{64} x^{4}+\frac{359}{32768} x^{5}-\frac{75}{32768} x^{6}+\frac{919}{2097152} x^{7}+\ldots
$$

The points $\xi_{k N}:=\xi\left(x_{k N}\right)$, for $x_{k N}$ small, are true poles of $y(x)$.

The full description of the poles distribution on the universal covering of $\mathbf{C} \backslash\{0,1, \infty\}$ for the Hitchin solutions is given in [1], where two sequences of poles are determined. Hitchin solutions are solution of the Painlevé VI equation with coefficients $\alpha=\beta=\gamma=1 / 8$ and $\delta=3 / 8$, and their image through an Okamoto's transformation (see [23] and also [7]) is the Picard solutions. This transformation annihilates one sequence of poles, and conserves the other, which is given by a simple formula in terms of Theta functions. For $x$ small, this formula coincides with the $\xi_{k N}$ determined here by local analysis.

\section{The General PVI}

According to [14], the general PVI admits solutions with expansion (1) and coefficients

$$
y_{n}(x)=\sum_{m=-n}^{n} A_{n m}(\alpha, \beta, \gamma, \delta) e^{2 i m d} x^{2 i m \nu}
$$

The coefficients, as algebraic functions of $\alpha, \beta, \gamma, \delta$, can be computed by the procedure of [14]. For example:

$$
A_{1, \pm 1}=\frac{\alpha+2 \nu^{2} \mp \sqrt{\gamma\left(2 \alpha-4 \nu^{2}-\gamma\right)}}{8 \nu^{2}}, \quad A_{10}=-\frac{\alpha-2 \nu^{2}-\gamma}{4 \nu^{2}} .
$$

(Note that it is allowed the freedom $A_{11} \mapsto c A_{11}$ and $A_{1,-1} \mapsto c^{-1} A_{1,-1}, c \in \mathbf{C} \backslash\{0\}$, which is equivalent to a redefinition of $d$. But $c e^{2 i d}$ is fixed by the monodromy data). Theorem 1 holds, namely $y_{1}(x)$ has two infinite sequences of zeros which accumulate at $x=0$ along rays, accoding to the formula

$$
x_{k}(j)=\exp \left\{-\frac{d}{\nu}-\frac{i}{2 \nu} \ln \left[(-)^{j} \sqrt{\frac{A_{10}^{2}}{4 A_{11}^{2}}-\frac{A_{1,-1}}{A_{11}}}-\frac{A_{10}}{2 A_{11}}\right]-\frac{k \pi}{\nu}\right\}, \quad k \in \mathbf{N}, \quad j=1,2
$$

The argument of the logarithm is fixed once and for all. The poles of $y(x)$ asymptotically approach these zeros, as their absolute value tends to zero, with a series of the form

$$
\xi_{k}(j)=x_{k}(j)+\sum_{N=2}^{\infty} \Delta_{N}(j) x_{k}(j)^{N}, \quad k \rightarrow+\infty, \quad x_{k}(j) \rightarrow 0 .
$$

Also Theorem 2 holds.

\section{Proof of Theorem 1}

The formula for the zeros $x_{k}(j)$ is proved by solving

$$
y_{1}(x)=\frac{(2 \mu-1+2 i \nu)^{2} e^{-2 i d} x^{-2 i \nu}}{16 \nu^{2}}-\frac{(2 \mu-1)^{2}-4 \nu^{2}}{8 \nu^{2}}+\frac{(2 \mu-1-2 i \nu)^{2} e^{2 i d} x^{2 i \nu}}{16 \nu^{2}}=0
$$

Let $x_{k}(j)$ be one of the zeros so obtained. In Theorem 2 (whose prove is independent of the following and can be done first) we prove that there exists $\epsilon>0$ small such that there are no 
zeros in $\mathcal{U}\left(\left|x_{k}(j)\right|, \epsilon\right)$ of figure 3. Thus, it makes sense to look for the zero $\xi_{k}(j)$ of $y(x)$ closest to $x_{k}(j)$. Because $\epsilon$ is proportional to $\left|x_{k}(j)\right|$, the lenghts of the arc between $x_{k}(j)$ and $x_{k}(j) e^{ \pm i \epsilon}$ is proportional to $\left|x_{k}(j)\right|^{2}$. So, one expects that $\xi_{k}(j)=x_{k}(j)+O\left(x_{k}(j)^{2}\right)$. Therefore, we look for a zero of the form:

$$
\xi_{k}(j)=x_{k}(j)+\Delta, \quad \Delta=\Delta(x):=\sum_{n=1}^{\infty} \Delta_{n+1} x^{n+1}, \quad \Delta_{n+1} \in \mathbf{C} .
$$

Let $Y(x)=\sum_{n=1}^{\infty} x^{n-1} y_{n}(x)$. Let $k$ be greater than $k_{0}$, where $k_{0}$ is the minimum value such that $x_{k_{0}}(j)$ lies in the domain of convergence of (1). Impose:

$$
0=Y\left(x_{k}(j)+\Delta\right)=\sum_{n=1}^{\infty}\left(x_{k}(j)+\Delta\right)^{n-1} y_{n}\left(x_{k}(j)+\Delta\right)
$$

The series converges if $x_{k}(j)$ and $x_{k}(j)+\Delta$ lie in the domain of convergence of (1). Then, expand:

$$
y_{n}\left(x_{k}(j)+\Delta\right)=\left.\sum_{m=0}^{\infty} \frac{1}{m !} \frac{d^{m} y_{n}}{d x^{m}}\right|_{x_{k}(j)} \Delta^{m}
$$

Observe that $\left[x_{k}(1)\right]^{2 i \nu}=e^{-2 i d},\left[x_{k}(2)\right]^{2 i \nu}=\left(\frac{2 \mu-1+2 i \nu}{2 \mu-1-2 i \nu}\right)^{2} e^{-2 i d}$. It follows that the derivative of $y_{n}(x)=\sum_{m=-n}^{m} A_{n m}(\nu, \mu) e^{2 i m d} x^{2 i m \nu}$ computed at $x_{k}(1)$ or $x_{k}(2)$ has the structure:

$$
\left.\frac{d^{N} y_{n}}{d x^{N}}\right|_{x=x_{k}(j)}=\frac{Y_{n N}^{(j)}}{\left[x_{k}(j)\right]^{N}}, \quad j=1,2
$$

where $Y_{n N}^{(j)}$ are constants which do not depend on $k$. They depend only on $\nu, \mu$. In particular, $Y_{n 0}^{(j)}=y_{n}\left(x_{k}(j)\right)$, and $Y_{10}^{(j)}=y_{1}\left(x_{k}(j)\right)=0$. We have therefore to determine $\Delta=\Delta(x)$ which solves:

$$
0=\sum_{n=1}^{\infty}\left[(x+\Delta)^{n-1} \sum_{m=0}^{\infty} \frac{Y_{n m} \Delta^{m}}{m ! x^{m}}\right]=\sum_{n=1}^{\infty} x^{n-1}\left[(1+\tilde{\Delta})^{n-1} \sum_{m=0}^{\infty} \frac{Y_{n m}}{m !} \tilde{\Delta}^{m}\right], \quad \tilde{\Delta}=\frac{\Delta}{x}
$$

This is similar to a problem of reversion of a series, though it is not in the form which allows to apply Lagrange inversion theorem to find $\tilde{\Delta}(x)$. Nevertheless, the coefficients are computable by putting equal to zero the coefficients of the powers of $x$ in the series expansion (omitting $k$ and $j$ ):

$$
\begin{gathered}
0=\sum_{n=1}^{\infty}\left[x^{n-1}\left(1+\sum_{l=1}^{\infty} \Delta_{l+1} x^{l}\right)^{n-1}\left(\sum_{m=0}^{\infty} \frac{Y_{n m}}{m !}\left(\sum_{l=1}^{\infty} \Delta_{l+1} x^{l}\right)^{m}\right)\right] \\
=\sum_{n=1}^{\infty}\left[Y_{n 0}+\sum_{p=1}^{n}\left(\begin{array}{c}
n-1 \\
p-1
\end{array}\right) \sum_{m=1}^{\infty} \frac{Y_{n m}}{m !} \sum_{l_{1}, \ldots, l_{m+p-1}=1}^{\infty} \Delta_{l_{1}+1} \ldots \Delta_{l_{m+p-1}+1} x^{l_{1}+\ldots l_{m+p-1}}\right] x^{n-1}
\end{gathered}
$$

The above series determines all the $\Delta_{n}$ 's recursively, provided that $Y_{10}=0$, namely provided that $x_{k}(j)$ is a zero of $y_{1}(x)$. The first terms are:

$$
\begin{gathered}
\Delta_{2}=-\frac{Y_{20}}{Y_{11}}, \quad \Delta_{3}=-\frac{Y_{30}}{Y_{11}}+\frac{2 Y_{20}\left(Y_{20}+Y_{21}\right) Y_{11}-Y_{12} Y_{20}^{2}}{2 Y_{11}^{3}} \\
\Delta_{4}=-\frac{Y_{40}}{Y_{11}}+\frac{1}{2 Y_{11}^{5}}\left\{\left[\left(6 Y_{30}+2 Y_{31}\right) Y_{20}+2 Y_{21} Y_{30}\right] Y_{11}^{3}+\right.
\end{gathered}
$$




$$
\begin{gathered}
-2\left(Y_{20}^{2}+\left(3 Y_{21}+\frac{1}{2} Y_{22}\right) Y_{20}+Y_{12} Y_{30}+Y_{21}^{2}\right) Y_{20} Y_{11}^{2}+ \\
\left.+\left(\left(3 Y_{12}+\frac{1}{3} Y_{13}\right) Y_{20}^{3}+3 Y_{12} Y_{20}^{2} Y_{21}\right) Y_{11}-Y_{12}^{2} Y_{20}^{3}\right\}
\end{gathered}
$$

The above formulas do not hold for double zeros, namely $Y_{11}=0$, which occur for $\mu=\frac{1}{2}$. In this case, the procedure of inversion of the series works if also $Y_{20}=0$, which is true when $\mu=\frac{1}{2}$. The formulas obtained are more complicated and will be omitted here. The case $\mu=\frac{1}{2}$ corresponds to a sub-class of Picard solutions, and it is solved in Section 4.

Note: The computation, rather complicated, can be done on a computer. To determine $\Delta_{2}$, $\Delta_{3}, \ldots \Delta_{N+1}$ one can write the following expression for a program of symbolic computation like Maple or Mathematica:

$$
0=\sum_{n=1}^{N+1}\left[\left(x+\sum_{l=1}^{N} \Delta_{l+1} x^{l+1}\right)^{n-1}\left(\sum_{m=0}^{N} \frac{Y_{n m}\left(\sum_{l=1}^{N} \Delta_{l+1} x^{j+1}\right)^{m}}{m ! x^{m}}\right)\right]
$$

It is enough to compute $y_{1}$ up the $N$-th derivatives, $y_{2}$ up to order $(N-1)$-th derivative,..., $y_{N-1}$ up to first derivative, and $y_{N}$.

We compute the $Y_{n N}^{(j)}$ 's from the $A_{n m}$ 's, and find $Y_{11}^{(1)}=-Y_{11}^{(2)}=2 \mu-1, Y_{20}^{(1)}=-Y_{20}^{(1)}=\mu-\frac{1}{2}$. It follows that $\Delta_{2}(1)=\Delta_{2}(2)=-\frac{1}{2}$. Higher order computations, up to $N=3$, give the other formulas of $\Delta_{N}(j)$ stated in the Theorem. Note that, though the derivation of the result requires $\mu \neq \frac{1}{2}$, these formulas have limit for $\mu \rightarrow \frac{1}{2}$, which coincide with the result of the example of Section 4 .

It is necessary to show that the result is consistent. Observe that $\xi_{k}(j)=x_{k}(j)-\frac{1}{2} x_{k}(j)^{2}+$ $O\left(x_{k}(j)^{3}\right)$. Thus, we need to show that there exist a $K$ such that for any $k \geq K$ a disk of radius $\left|x_{k}(j)\right|^{2}$ with center $x_{k}(j)$ does not intersect a similar disk around another zero. This would imply that for any $j=1,2$ and for any $k \geq K$, equation (30) has a unique formal solution $x_{k}(j)+\Delta^{(j)}\left(x_{k}(j)\right)$, with $\Delta^{(j)}(x)=\sum_{N \geq 2} \Delta_{N}(j) x^{N}$, where the $\Delta_{N}(j)$ 's have been uniquely constructed by the procedure above. We distinguish two orderings of the zeros

- When the ordering is $\left|x_{k+1}(1)\right|<\left|x_{k}(2)\right|<\left|x_{k}(1)\right|$. Two cases must be considered: i) $x_{k}(1)$ and $x_{k}(2)$; ii) $x_{k}(2)$ and $x_{k+1}(1)$. In case i), one must check if the following holds:

$$
\left|x_{k}(2)\right|+\left|x_{k}(2)\right|^{2}<\left|x_{k}(1)\right|-\left|x_{k}(1)\right|^{2}
$$

Since $\left|x_{k}(2)\right|=e^{\frac{\theta}{\nu}}\left|x_{k}(1)\right|$, where $-\pi<\theta=\arg \left(\frac{2 \mu-1+2 i \nu}{2 \mu-1-2 i \nu}\right) \leq 0$, the above becomes:

$$
\left|x_{k}(1)\right| e^{\frac{\theta}{\nu}}+\left|x_{k}(1)\right|^{2} e^{\frac{2 \theta}{\nu}}<\left|x_{k}(1)\right|-\left|x_{k}(1)\right|^{2}
$$

which holds for

$$
0<\left|x_{k}(1)\right|<\frac{1-e^{\frac{\theta}{\nu}}}{1+e^{\frac{2 \theta}{\nu}}}<1
$$

In case ii), one must check if the following holds:

$$
\left|x_{k+1}(1)\right|+\left|x_{k+1}(1)\right|^{2}<\left|x_{k}(2)\right|-\left|x_{k}(2)\right|^{2}
$$

Since $\left|x_{k+1}(1)\right|=e^{-\frac{\pi+\theta}{\nu}}\left|x_{k}(2)\right|$, the above becomes:

$$
\left|x_{k}(2)\right| e^{-\frac{\pi+\theta}{\nu}}+\left|x_{k}(2)\right|^{2} e^{-\frac{2(\pi+\theta)}{\nu}}<\left|x_{k}(2)\right|-\left|x_{k}(2)\right|^{2}
$$


which holds for

$$
0<\left|x_{k}(2)\right|<\frac{1-e^{-\frac{\pi+\theta}{\nu}}}{1+e^{-\frac{2(\pi+\theta)}{\nu}}}<1
$$

Therefore, there exist $K$ big enough such that for any $k \geq K$ the above inequalities i) and ii) are satisfied.

- When the ordering is $\left|x_{k}(1)\right|<\left|x_{k}(2)\right|<\left|x_{k-1}(1)\right|$. Two cases must be considered: i) $x_{k-1}(1)$ and $x_{k}(2)$; ii) $x_{k}(2)$ and $x_{k}(1)$. We proceed and conclude in a similar way that there exist $K$ big enough such that for any $k \geq K$ the inequalities are satisfied.

\section{Proof of Theorem 2}

The question to be answered is where the zeros of $\frac{1}{y(x)}=\sum_{n=1}^{\infty} x^{n-1} y_{n}(x)$ do not lie. First, note that $x=0$ is an essential singularity for $y_{1}(x)$ (with $\left|y_{1}(x)\right|$ bounded), and it is not a zero. Let us write:

$$
\sum_{n=1}^{\infty} x^{n-1} y_{n}(x)=y_{1}(x)+x f(x)
$$

where $f(x)$ is a bounded function in a disk of radius smaller than the radius of convergence of the series above. Let again $k \geq k_{0}$, where $k_{0}$ is such that $x_{k_{0}}(j)$ is the biggest zero in the domain of convergence. $j$ is either $j=1$ or $j=2$, depending on the order $\left|x_{k}(2)\right|<\left|x_{k}(1)\right|$ or $\left|x_{k}(1)\right|<\left|x_{k}(2)\right|$, the first case occurring when $-\pi<\arg \left(\frac{2 \mu-1+2 i \nu}{2 \mu-1-2 i \nu}\right) \leq 0$, and the second when $0<\arg \left(\frac{2 \mu-1+2 i \nu}{2 \mu-1-2 i \nu}\right) \leq \pi$. Boundedness of $f(x)$ means that there exists $C_{f}>0$ such that $|f(x)| \leq C_{f}$ for any $x$ in the disk $|x| \leq\left|x_{k_{0}}(j)\right|$.

First, we prove the statement for the case $\mu=-1$. The zeros of $y_{1}(x)$ are on the negative immaginary axis and $x=0$. For any $0<\epsilon<\pi, y_{1}(x)$ is bounded and not vanishing in the domain $\mathcal{U}\left(\left|x_{k_{0}}(1)\right|, \epsilon\right)$ of the type represented in figure 3. Namely, for any $\epsilon>0$ small, there exist $C_{\epsilon}>0$ such that $\left|y_{1}(x)\right| \geq C_{\epsilon}$ for $x \in \mathcal{U}\left(\left|x_{k_{0}}(1)\right|, \epsilon\right)$. Observe that $C_{\epsilon} \rightarrow 0$ as $\epsilon \rightarrow 0$, because $x$ may take values closer to the zeros of $y_{1}(x)$ as $\epsilon$ gets smaller. The following simple estimate holds for $x \in \mathcal{U}\left(\left|x_{k_{0}}(1)\right|, \epsilon\right)$ :

$$
\left|y_{1}(x)+x f(x)\right| \geq|| y_{1}(x)|-| x|| f(x)|| \geq\left|y_{1}(x)\right|-|x| C_{f} \geq C_{\epsilon}-|x| C_{f}
$$

We have:

$$
C_{\epsilon}-|x| C_{f}>0 \Longleftrightarrow|x|<\frac{C_{\epsilon}}{C_{f}}
$$

Namely, $y_{1}(x)+x f(x)$ has no zeros in $\mathcal{U}\left(R_{\epsilon}, \epsilon\right)$, for any $0<R_{\epsilon}<\frac{C_{\epsilon}}{C_{f}}$.

In order to prove the estimate $C_{\epsilon}=3 \tan (\epsilon)\left(1+O\left(\tan (\epsilon)^{2}\right)\right)$ for $\epsilon \rightarrow 0$, we evaluate $\left|y_{1}(x)\right|$ along the two rays of angle $-\frac{\pi}{2} \pm \epsilon$, which are the boundary of the sectorial cut of $\mathcal{U}\left(\left|x_{k_{0}}(1)\right|, \epsilon\right)$. On these rays $\left|y_{1}(x)\right|$ take minimal values as $\epsilon \rightarrow 0$, because the zeros on the negative immaginary axis are approached. We write

$$
y_{1}(x)=\frac{(3-2 i \nu)^{2}}{16 \nu^{2}} e^{-2 i d} x^{-2 i \nu}-\frac{9-4 \nu^{2}}{8 \nu^{2}}+\frac{(3+2 i \nu)^{2}}{16 \nu^{2}} e^{2 i d} x^{2 i \nu}
$$




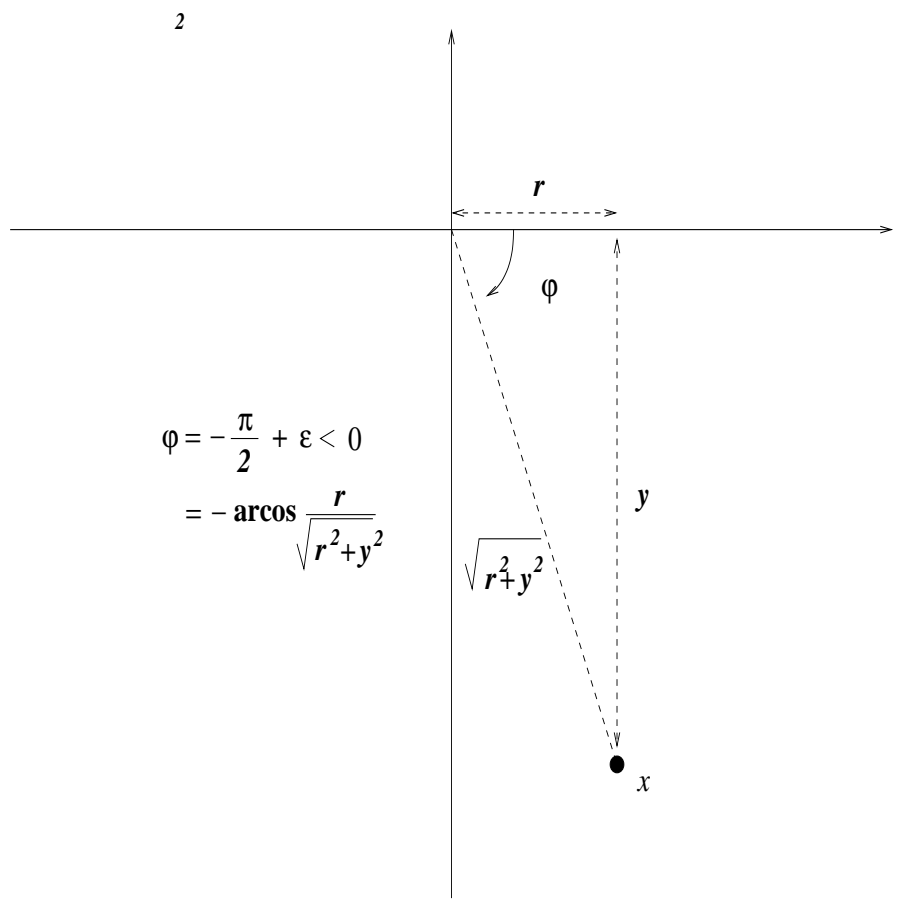

Figure 10:

We consider the ray of angle $-\frac{\pi}{2}+\epsilon$, the other being analogous. Let us write $(3 \pm 2 i \nu)^{2}=$ $\left(9+4 \nu^{2}\right) \exp \{ \pm 2 i \psi\}$, where $\psi=\operatorname{arcos}\left(\frac{3}{\sqrt{9+4 \nu^{2}}}\right)>0$. Let us also write $x=r-i w, w>0$ along the ray (see figure 10). Thus:

$$
(r-i w)^{2 i \nu}=e^{2 \nu \arccos \left(\frac{r}{\sqrt{r^{2}+w^{2}}}\right)} e^{i \nu \ln \left(r^{2}+w^{2}\right)}
$$

where $\arccos \left(\frac{r}{\sqrt{r^{2}+w^{2}}}\right)=\frac{\pi}{2}-\epsilon>0$. Recall that $\Im d=\frac{\pi}{2} \nu$. It follows that:

$$
\begin{gathered}
\left|y_{1}(x)\right|^{2}=\left(\frac{9+4 \nu^{2}}{8 \nu^{2}}\right)^{2}\left\{\left(\cosh (a) \cos (b)-\frac{9-4 \nu^{2}}{9+4 \nu^{2}}\right)^{2}+\sinh (a)^{2} \sin (b)^{2}\right\} . \\
a=\nu\left(\pi-2 \arccos \frac{r}{\sqrt{r^{2}+w^{2}}}\right), \quad b=\nu \ln \left(r^{2}+w^{2}\right)+2(\psi+\Re d) .
\end{gathered}
$$

When $\epsilon \rightarrow 0$, then $r \rightarrow 0$. The minimal values are obtained when $w=\left|x_{k}(j)\right|, k \in \mathbf{N}, j=1,2$, where $x_{k}(j)$ is a zero of $y_{1}(x)$. To this end, before proceeding, observe that if we let $r \rightarrow 0$ with $w \neq 0$ fixed, then $-\arccos \frac{r}{\sqrt{r^{2}+w^{2}}} \rightarrow-\frac{\pi}{2}$ and $a \rightarrow 0$. Therefore:

$$
\left|y_{1}(x)\right|^{2} \rightarrow\left(\frac{9+4 \nu^{2}}{8 \nu^{2}}\right)^{2}\left(\cos b-\frac{9-4 \nu^{2}}{9+4 \nu^{2}}\right)^{2}, \quad b=2 \nu \ln w+2(\psi+\Re d) .
$$

This takes minimal value $=0$ if:

$$
b=\arccos \frac{9-4 \nu^{2}}{9+4 \nu^{2}}-2 k \pi, \quad k \in \mathbf{N},
$$


namely:

$$
w=\exp \left\{-\frac{\Re d}{\nu}-\frac{\psi}{\nu}+\frac{1}{2 \nu} \arccos \frac{9-4 \nu^{2}}{9+4 \nu^{2}}\right\} \exp \left\{-k \frac{\pi}{\nu}\right\} .
$$

We prove that the above coincides with the zeros of $y_{1}(x)$. To see it, observe that:

$$
\begin{gathered}
0<\psi=\arccos \frac{3}{\sqrt{9+4 \nu^{2}}}=-i \ln \left(\frac{3+2 i \nu}{\sqrt{9+4 \nu^{2}}}\right) \\
\arccos \frac{9-4 \nu^{2}}{9+4 \nu^{2}}=-i \ln \left(\frac{9-4 \nu^{2}}{9+4 \nu^{2}} \pm \sqrt{\left(\frac{9-4 \nu^{2}}{9+4 \nu^{2}}\right)^{2}-1}\right)=-2 i \ln \left(\frac{3 \pm 2 i \nu}{\sqrt{9+4 \nu^{2}}}\right)
\end{gathered}
$$

which implies:

$$
-\frac{\psi}{\nu}+\frac{1}{2 \nu} \arccos \frac{9-4 \nu^{2}}{9+4 \nu^{2}}=-\frac{i}{\nu} \ln \left(\frac{3 \pm 2 i \nu}{3+2 i \nu}\right)
$$

and then (33) corresponds to the (absolute value of the) zeros:

$$
\left|x_{k}(j)\right|=\exp \left\{-\frac{\Re d}{\nu}+\frac{j-1}{\nu} \arg \frac{3-2 i \nu}{3+2 i \nu}\right\} \exp \left\{-k \frac{\pi}{\nu}\right\}, \quad j=1,2
$$

Now we proceed to the estimate of $y_{1}(x)$ along the radius of angle $-\frac{\pi}{2}+\epsilon$, when $r \rightarrow 0$. We have:

$$
0<\arccos \frac{r}{\sqrt{r^{2}+w^{2}}}=-i \ln \left(\frac{r+i w}{\sqrt{r^{2}+w^{2}}}\right)=\frac{\pi}{2}-\frac{r}{w}+O\left(\frac{r^{3}}{w^{3}}\right)
$$

Thus $a=\frac{2 \nu r}{w}+O\left(\frac{r^{3}}{w^{3}}\right)$, and

$$
\cosh (a)=1+\frac{2 \nu^{2} r^{2}}{w^{2}}+O\left(\frac{r^{3}}{w^{3}}\right), \quad \sinh (a)=\frac{2 \nu r}{w}+O\left(\frac{r^{3}}{w^{3}}\right)
$$

Moreover:

$$
\begin{gathered}
b=\nu \ln \left(r^{2}+w^{2}\right)+2(\psi+\Re d)=\nu\left[\ln \left(w^{2}\right)+\ln \left(1+\frac{r^{2}}{w^{2}}\right)\right]+2(\psi+\Re d) \\
=2 \nu \ln w+2(\psi+\Re d)+\frac{\nu r^{2}}{w^{2}}+O\left(\frac{r^{4}}{w^{4}}\right)
\end{gathered}
$$

The evaluation will be done when, for $\epsilon$ small and $r$ small, the position of $r-i w$ is close to a zero of $y_{1}(x)$, namely to a minimum of $\left|y_{1}(x)\right|$. According to the above computations for $r=0$ and $w$ fixed, we have that $r-i w$ is close to a zero of $y_{1}(x)$ when $w=\left|x_{k}(j)\right|$. Namely:

$$
2 \nu \ln w+2(\psi+\Re d)=\arccos \frac{9-4 \nu^{2}}{9+4 \nu^{2}}-2 k \pi
$$

( $j=1,2$ is given by the to signs of arccos). Therefore, for small $r$ and $w=\left|x_{k}(j)\right|$ :

$$
\cos (b)=\cos \left(\arccos \frac{9-4 \nu^{2}}{9+4 \nu^{2}}+\frac{\nu r^{2}}{w^{2}}+O\left(\frac{r^{4}}{w^{4}}\right)\right) .
$$


Now, use the fact that $\cos (\theta+\delta)=\cos \theta-\delta \sin \theta-\frac{\delta^{2}}{2} \cos \theta+O\left(\delta^{3}\right)$ when $\delta \rightarrow 0$, to obtain:

$$
\begin{gathered}
\cos (b)=\frac{9-4 \nu^{2}}{9+4 \nu^{2}}-\frac{\nu r^{2}}{w^{2}} \sqrt{1-\left(\frac{9-4 \nu^{2}}{9+4 \nu^{2}}\right)^{2}}+O\left(\frac{r^{4}}{w^{4}}\right)=\frac{9-4 \nu^{2}}{9+4 \nu^{2}}-\frac{12 \nu^{2}}{9+4 \nu^{2}} \frac{r^{2}}{w^{2}}+O\left(\frac{r^{4}}{w^{4}}\right) \\
0<\sin (b)=\sqrt{1-\cos (b)^{2}}=\frac{12 \nu}{9+4 \nu^{2}}\left(1-\frac{9-4 \nu^{2}}{12} \frac{r^{2}}{w^{2}}+O\left(\frac{r^{4}}{w^{4}}\right)\right)
\end{gathered}
$$

Composing all the above expansions into (32), we finally obtain the minimal values:

$$
\left|y_{1}(x)\right|=\frac{3 r}{w}\left(1+O\left(\frac{r^{2}}{w^{2}}\right)\right)=3 \tan (\epsilon)\left(1+O\left(\tan (\epsilon)^{2}\right)\right)
$$

We turn to the general case. The domain $\mathcal{U}\left(\left|x_{k_{0}}(j)\right|, \epsilon\right)$ is that of figure 3 . There is no conceptual change in proving the inequality (31). The estimate of $C_{\epsilon}$ is done as for the case $\mu=-1$, for both the sector cuts of $\mathcal{U}\left(\left|x_{k_{0}}(j)\right|, \epsilon\right)$. Technically, it is more complicated, but methodologically the same. The final result is that the minimal values of $\left|y_{1}(x)\right|$ are

$$
\left|y_{1}(x)\right|=\frac{|2 \mu-1| r}{w}\left(1+O\left(\frac{r^{2}}{w^{2}}\right)\right)=|2 \mu-1| \tan (\epsilon)\left(1+O\left(\tan (\epsilon)^{2}\right)\right)
$$

Thus $C_{\epsilon}=|2 \mu-1| \tan (\epsilon)\left(1+O\left(\tan (\epsilon)^{2}\right)\right), \epsilon \rightarrow 0$. In the case $\mu=\frac{1}{2}$, the above procedure should be applied expanding $\left|y_{1}\right|$ at least to order $r^{2} / w^{2}$, because the zeros of $y_{1}(x)$ are double $\left(x_{k+1}(1)=x_{k}(2)\right)$, and $\frac{d^{2} y_{1}\left(x_{k}(j)\right)}{d x^{2}}=0$. The final result is

$$
\left|y_{1}(x)\right|=\frac{2 \nu^{2} r^{2}}{w^{2}}\left(1+O\left(\frac{r^{3}}{w^{3}}\right)\right)=2 \nu^{2} \tan (\epsilon)^{2}\left(1+O\left(\tan (\epsilon)^{3}\right)\right)
$$

\section{Proof of Proposition 1:}

The parametrization of a branch (1) in terms of monodromy data is computed in section 6 of our [14] (see also Proposition 6 of [14]), where we find the notation $\sigma_{0}=1+2 i \nu$ and the result $2 \cos \pi \sigma_{0}=p_{0 x}$. Thus, $\nu$ is determined by $\cosh (2 \pi \nu)=-\frac{p_{0 x}}{2}$, which has solutions:

$$
\exp \{ \pm 2 \pi \nu\}= \pm \frac{1}{2} \sqrt{p_{0 x}^{2}-4}-\frac{p_{0 x}}{2}, \quad \nu>0
$$

This proves (12). In [14] $d$ is computed, and the result is:

$$
d=\frac{i}{2} \ln \left\{\frac{8 i \nu r}{(2 \nu+i(1-2 \mu))^{2}}\right\}
$$

where

$$
r=-\frac{16^{2 i \nu} \Gamma\left(\frac{3}{2}-\frac{\theta_{\infty}}{2}-i \nu\right)^{2} \Gamma\left(\frac{\theta_{\infty}}{2}+\frac{1}{2}-i \nu\right)^{2}}{2 i \nu^{3} \sinh (2 \pi \nu)^{2} \Gamma(-i \nu)^{4}} \times
$$




$$
\times\left[\frac{1}{2}\left(e^{2 \pi \nu} p_{x 1}-p_{01}\right) \sinh (2 \pi \nu)+\left(\cos \left(\pi \theta_{\infty}\right)+1\right)\left(e^{2 \pi \nu}+1\right)\right] .
$$

This proves (13). In the special cases $2 \mu= \pm 2 i \nu+2 m+1$, we have $p_{\infty}=p_{0 x}$. The affine cubic becomes a polynomial of degree 2 in $p_{01}$, with to solutions $p_{01}=2-2 e^{ \pm 2 \pi \nu}+p_{x 1} e^{ \pm 2 \pi \nu}$. The formulae for $d$ when $2 \mu=2 i \nu+1-2 m, m=-1,-2,-3, \ldots$, and $2 \mu=-2 i \nu+2 m-1$, $m=0,-1,-2,-3, \ldots$, can be obtained by substitution into the formula of the generic case. This is not possible in other cases, where we need the formulas computed in our [10]. In [10] we find the Jimbo's solution

$$
y_{[10]}(x) \sim a x^{1-\sigma}, \quad 0<\Re \sigma<1
$$

whose coefficient $a$ is computed in terms of monodromy data also in the special cases $\sigma \pm 2 \mu=$ $2 m$. Solution (1) can be ritten as:

$$
y(x)=\frac{1}{A_{1,-1} e^{-2 i d} x^{1-\sigma}+A_{10}+A_{11} e^{2 i d} x^{\sigma-1}+O(x)}, \quad \sigma=1+2 i \nu
$$

Now, let $\sigma=1+2 i \nu-\varepsilon, 0<\varepsilon \rightarrow 0$, and rewrite

$$
y(x)=\frac{A_{11}^{-1} e^{-2 i d} x^{1-\sigma}}{1+\text { higer order corrections } O\left(x^{\varepsilon}\right)}
$$

Then, identify $A_{11}^{-1} e^{-2 i d}=a$ ( $a$ is given in theorem 2, page 301, of [10]), extract $d$ and let $\varepsilon \rightarrow 0$. This completes the proof. (Note that in [10], the notations of [6] are used, namely $x_{0}^{2}=2-p_{0 x}, x_{1}^{2}=2-p_{x 1}$ and $\left.x_{\infty}^{2}=2-p_{01}\right)$.

\section{Proof of theorem 3 and its Corollary}

\subsection{The case $p_{1 x}=p_{01}=p_{0 x}$}

The behavior at $x=0$ of a branch of a $P V I_{\mu}$-transcendent is explicitly parameterized by the monodromy data $\theta_{\infty}=2 \mu$ (i.e. $p_{\infty}$ ), $p_{0 x}, p_{01}, p_{x 1}$ to which it is in one-to-one correspondence. Let $\sigma$ be defined by $2 \cos \pi \sigma=p_{0 x}$ and $0 \leq \Re \sigma \leq 1$. Its value determines the critical behaviors as follows:

- When $0<\Re \sigma<1$, namely $p_{0 x} \notin(-\infty,-2] \cup[2,+\infty)$, the behavior is (see Jimbo [19]):

$$
y(x)=a x^{1-\sigma}\left(1+O\left(x^{\sigma}, x^{1-\sigma}\right)\right), \quad a=a\left(\sigma, \theta_{\infty}, p_{01}, p_{x 1}\right) \in \mathbf{C}
$$

- When $\sigma=2 i \nu, \nu>0$, namely $p_{0 x}>2$, the behavior is (see [19] and [14]):

$$
y(x)=x\left[\frac{(2 \mu-1+2 i \nu)^{2} e^{-2 i d} x^{-2 i \nu}}{16 \nu^{2}}-\frac{(2 \mu-1)^{2}-4 \nu^{2}}{8 \nu^{2}}+\frac{(2 \mu-1-2 i \nu)^{2} e^{2 i d} x^{2 i \nu}}{16 \nu^{2}}\right]+O\left(x^{2}\right)
$$

where $d=d\left(\nu, \theta_{\infty}, p_{01}, p_{x 1}\right) \in \mathbf{C}$.

- When $\sigma=1+2 i \nu$, namely $p_{0 x}<-2$, the behavior is (1).

In the special case $p_{0 x}=p_{x 1}=p_{01}$, the cubic surface (11) becomes the curve (21), depicted in figure 4 for $\cos \pi \theta_{\infty} \in \mathbf{R}$. It has three branches when $-1<\cos \pi \theta_{\infty}<1$, namely when $\theta_{\infty}$ is real. It has double points for $\cos \pi \theta_{\infty}=-1$, namely $\theta_{\infty}=2 m+1, m \in \mathbf{Z}$, and for $\cos \pi \theta_{\infty}=1$, 
namely $\theta_{\infty}=2 m, m \in \mathbf{Z}$. It has one branch when $\cos \pi \theta_{\infty}<-1$, namely $\theta_{\infty}=2 m+1+i \vartheta$, and when $\cos \pi \theta_{\infty}>1$, namely $\theta_{\infty}=2 m+i \vartheta, \vartheta>0$. We divide the curve into seven portions:

[i] The half-line for $\cos \pi \theta_{\infty}<-9$ and $p_{0 x}<-2$. Here $\sigma=1+2 i \nu, \nu>0$.

[ii] The point $\left(\cos \pi \theta_{\infty}, p_{0 x}\right)=(-9,-2)$. Here $\sigma=1$.

[iii] The segment of line connecting $(-9,-2)$ to $(1,2)$, where $-9<\cos \pi \theta_{\infty}<1$ and $-2<$ $p_{0 x}<2$. Here $0<\sigma<1$.

[iv] The point $\left(\cos \pi \theta_{\infty}, p_{0 x}\right)=(1,2)$. Here $\sigma=0$.

[v] the segment of line connecting $(-1,-2)$ and $(1,2)$, where $-1<\cos \pi \theta_{\infty}<1$ and $-2<$ $p_{0 x}<2$. Here $0<\sigma<1$.

[vi] The point $\left(\cos \pi \theta_{\infty}, p_{0 x}\right)=(-1,-2)$. Here $\sigma=1$.

[vii] The half-line for $\cos \pi \theta_{\infty}>-1$ and $p_{0 x}<-2$. Here $\sigma=1+2 i \nu, \nu>0$

The case of solutions (1), namely the case $\sigma=1+2 i \nu, \nu>0$, corresponds to the portions [i] and [vii] above. Included in this, is the case of the quantum cohomology of $C P^{2}$, which is on the portion [vii], for $\left(\cos \pi \theta_{\infty}, p_{0 x}\right)=(1,-7)$.

Lemma 1 Let $\sigma=1+2 i \nu, \nu>0, \nu=(2 / \pi) \ln G$. Let $p_{0 x}=p_{x 1}=p_{01}=2 \cos (\pi \sigma)=$ $-2 \cosh (2 \pi \nu) \leq-2$. The branch [i] $\cup[\mathrm{ii}]$ of the cubic curve (21) for $\left(\cos \pi \theta_{\infty}, p_{0 x}\right)$ between $(-\infty,-\infty)$ and $(-9,-2)$ has equations:

$$
\begin{aligned}
\cos \pi \theta_{\infty} & =-\frac{1}{2} e^{-3 \pi \nu}\left(3 e^{5 \pi \nu}+4 e^{3 \pi \nu}+3 e^{\pi \nu}+e^{6 \pi \nu}+3 e^{4 \pi \nu}+3 e^{2 \pi \nu}+1\right) \\
& =-\frac{\left(G^{2}+G+1\right)\left(G^{2}-G+1\right)\left(G^{8}+2 G^{6}+2 G^{2}+1\right)}{2 G^{6}}
\end{aligned}
$$

and:

$$
d=\frac{i}{2} \ln \left\{\frac{4\left(G^{4}+1\right)^{2}}{\left(G^{2}-1\right)^{2}} \frac{16^{2 i \nu} \Gamma\left(\frac{3-\theta_{\infty}}{2}-i \nu\right)^{2} \Gamma\left(\frac{1+\theta_{\infty}}{2}-i \nu\right)^{2}}{\nu^{2}\left(2 \nu+i\left(1-\theta_{\infty}\right)\right) \Gamma(-i \nu)^{4}}\right\}
$$

The branch [vii] $\cup[\mathrm{vi}]$ for $\left(\cos \pi \theta_{\infty}, p_{0 x}\right)$ between $(-1,-2)$ and $(+\infty,-\infty)$ has equations:

$$
\begin{gathered}
\cos \pi \theta_{\infty}=-\frac{1}{2} e^{-3 \pi \nu}\left(3 e^{5 \pi \nu}+4 e^{3 \pi \nu}+3 e^{\pi \nu}-e^{6 \pi \nu}-3 e^{4 \pi \nu}-3 e^{2 \pi \nu}-1\right) \\
=\frac{\left(G^{4}-G^{2}+1\right)\left(G^{8}-2 G^{6}-2 G^{2}+1\right)}{2 G^{6}}
\end{gathered}
$$

and:

$$
d=\frac{i}{2} \ln \left\{\frac{4\left(G^{4}+1\right)^{2}}{\left(G^{2}+1\right)^{2}} \frac{16^{2 i \nu} \Gamma\left(\frac{3-\theta_{\infty}}{2}-i \nu\right)^{2} \Gamma\left(\frac{1+\theta_{\infty}}{2}-i \nu\right)^{2}}{\nu^{2}\left(2 \nu+i\left(1-\theta_{\infty}\right)\right) \Gamma(-i \nu)^{4}}\right\}
$$

Proof: It is a matter of computation. To find (34) and (36) substitute $p_{0 x}=-2 \cosh (2 \pi \nu)$ in (21) and solve for $\cos \pi \theta_{\infty}$. Substitute $\nu=\frac{2}{\pi} \ln G$ into (34) and (36) and find (35) and (37). These last are then substituted in (13), together with $p_{1 x}=p_{01}=p_{0 x}=-2 \cosh (2 \pi \nu)$. Simple algebra gives the expression of $d$. 


\subsection{Proof of Theorem 3}

Let $\mu=-1$ and $p_{0 x}=p_{01}=p_{x 1}=-7$, so that the branch $y(x)$ in (1) is associated to the quantum cohomology of $C P^{2}$. Then, from (12) we immediately have $\nu=\frac{2}{\pi} \ln \frac{1+\sqrt{5}}{2}$. Substitution into (38) gives:

$$
d=\frac{i}{2} \ln \left\{\frac{4\left(\mathbf{G}^{4}+1\right)^{2}}{\left(\mathbf{G}^{2}+1\right)^{2}} \frac{16^{2 i \nu} \Gamma\left(\frac{5}{2}-i \nu\right)^{2} \Gamma\left(-\frac{1}{2}-i \nu\right)^{2}}{\nu^{2}(2 \nu+3 i) \Gamma(-i \nu)^{4}}\right\}
$$

Then, standard manipulation of $\Gamma$ functions proves (6). The second part of the theorem follows from theorem 1 . Please, note that we need already the result $\Im d=\frac{\pi \nu}{2}$ in order to compute the formula of the zeros. This is proved below.

\subsection{Proof of the Corollary}

We recall the following convergent expansion

$$
\ln (\Gamma(1+z))=-\gamma z-\frac{1}{2} \ln \left(\frac{\sin (\pi z)}{\pi z}\right)-\sum_{n=1}^{\infty} \frac{\zeta(2 n+1) z^{2 n+1}}{2 n+1}, \quad|z|<1
$$

It can be applied to the factors $\ln (\Gamma(1-2 i \nu))$ and $\ln (\Gamma(1-i \nu))$ in $(6)$, provided that $|\nu|<1 / 2$. We also need to fix the determination

$$
\ln \left(\frac{1-2 i \nu}{1+2 i \nu}\right)=-2 i \arccos \frac{1}{\sqrt{1+4 \nu^{2}}}
$$

with arccos positive for positive $\nu$ and zero for $\nu=0$. With this in mind, we expand (6) and find

$$
\begin{aligned}
d=\frac{\pi}{2}-8 \nu \ln (2)+2 \arccos & \frac{1}{\sqrt{1+4 \nu^{2}}}+4 \sum_{n=1}^{\infty} \frac{(-1)^{n}\left(1-4^{n}\right) \zeta(2 n+1)}{2 n+1} \nu^{2 n+1}+ \\
& +i \ln \left(\mathbf{G}^{2}-1\right)+k \pi,
\end{aligned}
$$

To conclude, observe that $\mathbf{G}^{2}-1=\mathbf{G}$, by definition of golden ratio. Thus $\ln \left(\mathbf{G}^{2}-1\right)=\ln \mathbf{G}=$ $\pi \nu / 2$.

\section{Proof of Proposition 4}

We distinguish two cases:

1) Generic case (13). To compute the expansion at $\nu=0$, we rewrite:

$$
\Gamma\left(\frac{3}{2}-\mu-i \nu\right) \Gamma\left(\frac{1}{2}+\mu-i \nu\right)=\frac{\pi\left(\frac{1}{2}-\mu-i \nu\right)}{\sin \left[\pi\left(\mu+\frac{1}{2}+i \nu\right)\right]} \frac{\Gamma\left(\frac{1}{2}+\mu-i \nu\right)}{\Gamma\left(\mu+\frac{1}{2}+i \nu\right)}
$$

The factor $\Gamma(z-x) / \Gamma(z+x), z=\mu+1 / 2, x=i \nu \rightarrow 0$, has Taylor series:

$$
\frac{\Gamma(z-x)}{\Gamma(z+x)}=1-2 \psi(z) x+2 \psi(z)^{2} x^{2}-\left(\frac{1}{3} \frac{d^{2} \psi(z)}{d z^{2}}+\frac{4}{3} \psi(z)^{3}\right) x^{3}+\ldots
$$


The other factors in (13) are easily expanded around $\nu=0$, and the statement of Proposition 4 follows.

2) Special cases (14), (15), (16), (17). We consider the case of (14), the others being analogous. Manipulation of $\Gamma$ functions gives:

$$
\frac{\nu^{2} \Gamma(m)^{2} \Gamma(2 i \nu+1-m)^{2}\left(p_{x 1}-2\right)}{16^{2 i \nu} \Gamma(1+i \nu)^{4}}=\frac{2-p_{x 1}}{4}\left[1+\sum_{k=1}^{m-1} \frac{4 i \nu}{k}+O\left(\nu^{2}\right)\right] e^{-8 i \nu \ln 2} \frac{\Gamma(1+2 i \nu)^{2}}{\Gamma(1+i \nu)^{4}}
$$

Recall that $\sum_{k=1}^{m-1} \frac{1}{k}=\psi(m)+\gamma$. Also, expand the exponential $e^{-8 i \nu \ln 2}$. We find:

$$
d=-\frac{i}{2} \ln \left(\frac{2-p_{x 1}}{4}\right)-\frac{i}{2} \ln \left(1+4 i \nu(\psi(m)+\gamma-2 \ln 2)+O\left(\nu^{2}\right)\right)-i \ln \frac{\Gamma(1+2 i \nu)}{\Gamma(1+i \nu)^{2}}
$$

The logarithm of $\Gamma$ functions is $O\left(\nu^{2}\right)$, as it is clear from the expansion (39). The statement of Proposition 4 follows.

Proposition 4 fails in the singularities $2 \mu=2 m+1, m \in \mathbf{Z}$, of the expansion of $d$ for $\nu \rightarrow 0$.

\section{Proof of Proposition 5}

Lemma 2 Let $\mathbf{G}$ be the golden ratio. On the segment of the curve (21) between $(-1,-2)$ and $(1,-7), \theta_{\infty}$ is real and $0 \leq \nu \leq \frac{2}{\pi} \ln \mathbf{G}$. As a function of $\nu, \theta_{\infty}$ is:

$$
\theta_{\infty}= \pm \frac{1}{\pi} \arg \left(c_{1}(\nu)-\frac{i}{2} \sqrt{c_{2}(\nu)}\right)+2 m+1, \quad m \in \mathbf{Z}
$$

where $c_{1}(\nu)$ and $c_{2}(\nu)$ are given by the expressions (22). In particular, $c_{2}(\nu) \geq 0$ and the square root is the positive one. Moreover $\left|c_{1}(\nu)-\frac{i}{2} \sqrt{c_{2}(\nu)}\right|=1$. The argument is chosen such that $-\pi \leq \arg \left(c_{1}(\nu)-\frac{i}{2} \sqrt{c_{2}(\nu)}\right) \leq 0$.

Proof: It is clear that $\theta_{\infty}$ is real, because $-1 \leq \cos \pi \theta_{\infty} \leq 1$. Solving (36) for $\theta_{\infty}$ we find:

$$
\theta_{\infty}=-\frac{i}{\pi} \ln \left(c_{1}(\nu) \pm \frac{i}{2} \sqrt{c_{2}(\nu)}\right)+2 m+1, \quad m \in \mathbf{Z}
$$

The discriminant $c_{2}(\nu)$ is positive for $-\frac{2}{\pi} \ln \mathbf{G}<\nu<\frac{2}{\pi} \ln \mathbf{G}$ and simple computation shows that in this case $\left|c_{1}(\nu)-\frac{i}{2} \sqrt{c_{2}(\nu)}\right|=1$. Therefore:

$$
\theta_{\infty}=\frac{1}{\pi} \arg \left(c_{1}(\nu) \pm \frac{i}{2} \sqrt{c_{2}(\nu)}\right)+2 m+1= \pm \frac{1}{\pi} \arg \left(c_{1}(\nu)-\frac{i}{2} \sqrt{c_{2}(\nu)}\right)+2 m+1
$$

Due to the symmetries of $P V I_{\mu}$, one can restrict to the case $-1 \leq \Re \mu<0$, i.e. $-2 \leq$ $\Re\left(\theta_{\infty}\right)<0$, and take

$$
\theta_{\infty}=-1+\frac{1}{\pi} \arg \left(c_{1}(\nu)-\frac{i}{2} \sqrt{c_{2}(\nu)}\right)
$$

In particular, for $\nu=\frac{2}{\pi} \ln \mathbf{G}\left(p_{0 x}=p_{x 1}=p_{01}=-7\right)$ :

$$
\left.c_{1}(\nu)-\frac{i}{2} \sqrt{c_{2}(\nu)}=-1 \quad \Longrightarrow \quad \theta_{\infty}=-2 \quad \text { (Quantum Cohomology of } C P^{2}, \mu=-1\right)
$$


For $\nu=0\left(p_{0 x}=p_{x 1}=p_{01}=-2\right)$ :

$$
\left.c_{1}(\nu)-\frac{i}{2} \sqrt{c_{2}(\nu)}=1 \quad \Longrightarrow \quad \theta_{\infty}=-1, \quad \text { (Chazy solutions of [22], } \mu=-\frac{1}{2}\right)
$$

Proof of Proposition 5: Observe that (41) implies:

$$
\begin{gathered}
\theta_{\infty}=-1+2 \omega(\nu), \quad \nu \rightarrow 0 \\
2 \omega(\nu):=-\pi \sqrt{3} \nu^{2}-\frac{\sqrt{3} \pi^{3}}{4} \nu^{4}-\frac{17}{120} \pi^{5} \sqrt{3} \nu^{6}+O\left(\nu^{8}\right)
\end{gathered}
$$

Substitute this into (38) and use the identities $\Gamma(z+1)=z \Gamma(z), \Gamma(1-z)=\pi \Gamma^{-1}(z) \sin ^{-1}(\pi z)$ to simplify. We obtain:

$$
d=\frac{i}{2} \ln \left(-\frac{\left(G^{4}+1\right)^{2} 16^{2 i \nu} \nu^{2} \Gamma^{2}(1-\omega-i \nu) \Gamma^{2}(1+\omega-i \nu)^{2}}{\left(G^{2}+1\right)^{2}(i \nu-\omega)^{2} \Gamma^{4}(1-i \nu)}\right), \quad G=e^{\frac{\pi \nu}{2}} .
$$

When $\nu \rightarrow 0$, then $|i \nu \pm \omega|<1$. Thus, one can use the expansion (39), applied to $\Gamma(1 \pm \omega-i \nu)$ and $\Gamma(1-i \nu)$. This gives the expansion of Proposition 5 .

\section{Appendix: Coefficients $A_{n m}$}

Order 3:

$$
\begin{gathered}
A_{33}=\frac{3}{2^{16}} \frac{(2 \mu-1-2 i \nu)^{6}}{\nu^{6}} \\
A_{32}=\frac{i}{2^{15}} \frac{(-2 \mu+2 i \nu+1)^{4}}{(-\nu+i)^{2} \nu^{6}} \times \\
\times\left(-128 \nu^{5}+288 i \nu^{4}+192 \nu^{3}+8 i(2 \mu+1)(2 \mu-3) \nu^{2}-9(i-2 \nu)(2 \mu-1)^{2}\right) \\
A_{31}=\frac{(-2 \mu+2 i \nu+1)^{2}}{2^{16} \nu^{6}(i-\nu)}\left(2048 \nu^{7}-560 i \nu^{4}-1616 \nu^{5}-1440 i \mu^{3}-1376 i \nu^{2} \mu-360 i \mu-840 \nu^{3}-3360 \nu^{3} \mu^{2}+\right. \\
+3360 \nu^{3} \mu+128 i \nu^{2} \mu^{4}+720 i \mu^{4}-256 i \nu^{2} \mu^{3}-2048 i \nu^{4} \mu^{2}+1504 i \nu^{2} \mu^{2}-45 \nu-720 \nu \mu^{4}+1440 \nu \mu^{3}+ \\
\left.-1080 \nu \mu^{2}+360 \nu \mu+336 i \nu^{2}+2048 i \nu^{4} \mu+45 i-3712 i \nu^{6}+1080 i \mu^{2}\right) \\
A_{30}=-\frac{(2 \mu-1)^{2}+4 \nu^{2}}{2^{14}\left(\nu^{2}+1\right)^{2} \nu^{6}}\left(320 \nu^{4} \mu^{4}+528 \nu^{2} \mu^{4}+240 \mu^{4}-640 \nu^{4} \mu^{3}-1056 \nu^{2} \mu^{3}-480 \mu^{3}+\right. \\
+448 \nu^{6} \mu^{2}+1328 \nu^{4} \mu^{2}+1224 \nu^{2} \mu^{2}+360 \mu^{2}-448 \nu^{6} \mu-1008 \nu^{4} \mu-696 \nu^{2} \mu+ \\
\left.-120 \mu-128 \nu^{8}-144 \nu^{6}+104 \nu^{4}+141 \nu^{2}+15\right)
\end{gathered}
$$

Order 4:

$$
\begin{gathered}
A_{44}=-\frac{(-2 \mu+2 i \nu+1)^{8}}{2^{20} \nu^{8}} \\
A_{43}=-\frac{i(-2 \mu+2 i \nu+1)^{6}}{2^{19}(\nu-i)^{2} \nu^{8}} \times \\
\times\left(-72 \nu^{5}+168 i \nu^{4}+120 \nu^{3}+12 i \nu^{2} \mu^{2}-12 i \nu^{2} \mu-21 i \nu^{2}+8 \nu+32 \nu \mu^{2}-32 \nu \mu-4 i-16 i \mu^{2}+16 i \mu\right)
\end{gathered}
$$




$$
\begin{gathered}
A_{42}=\frac{(-2 \mu+2 i \nu+1)^{4}}{2^{18}(\nu-i)^{2} \nu^{8}}\left(7+1120 i \nu^{5}-2976 i \nu^{7}-224 \mu^{3}+306 i \nu^{3}-56 \mu+336 i \nu \mu^{2}-112 i \nu \mu+\right. \\
-448 i \mu^{3} \nu+1264 i \nu^{3} \mu^{2}+512 i \nu^{5} \mu+32 i \mu^{4} \nu^{3}-64 i \nu^{3} \mu^{3}-512 i \mu^{2} \nu^{5}+224 i \mu^{4} \nu+ \\
\quad-1232 i \nu^{3} \mu-288 \nu^{2} \mu-64 \nu^{2} \mu^{4}+128 \nu^{2} \mu^{3}-1440 \nu^{4} \mu^{2}+224 \nu^{2} \mu^{2}+ \\
\left.+1440 \nu^{4} \mu+14 i \nu+112 \mu^{4}-3040 \nu^{6}+1024 \nu^{8}+168 \mu^{2}+76 \nu^{2}-200 \nu^{4}\right) \\
A_{41}=\frac{i(-2 \mu+2 i \nu+1)^{2}}{32^{19}(i-\nu)(\nu+i)^{2} \nu^{8}}\left(-84-36560 \nu^{7}-6504 i \nu^{5}-53248 i \nu^{9}+26880 \nu^{8} \mu+40704 \nu^{6} \mu^{3}+\right. \\
-20352 \mu^{4} \nu^{6}-8256 \nu^{2} \mu^{6}-4608 \nu^{4} \mu^{5}-26880 \mu^{2} \nu^{8}+24768 \nu^{2} \mu^{5}+1536 \mu^{6} \nu^{4}+13440 \mu^{3}+5376 i \mu^{6} \nu+ \\
+51264 i \nu^{7} \mu+3072 i \nu^{7} \mu^{4}+42768 i \mu^{4} \nu^{3}-48192 i \nu^{7} \mu^{2}+1008 \mu+59904 \nu^{6} \mu+16452 i \mu^{2} \nu^{3}-13440 \mu^{3} \nu+ \\
-34656 i \mu^{3} \nu^{3}+23616 i \nu^{5} \mu+24576 i \nu^{9} \mu+10176 i \nu^{3} \mu^{6}-19200 i \nu^{5} \mu^{3}-16128 i \mu^{5} \nu-80256 \nu^{6} \mu^{2}+ \\
-37632 \nu^{4} \mu^{4}+82944 \nu^{4} \mu^{3}+12492 \nu^{2} \mu-52848 \nu^{2} \mu^{4}+64416 \nu^{2} \mu^{3}-86688 \nu^{4} \mu^{2}-40572 \nu^{2} \mu^{2}+44448 \nu^{4} \mu+ \\
+447 i \nu^{3}+84 i \nu-23552 i \nu^{11}-20160 \mu^{4}-12552 \nu^{6}+5040 i \mu^{2} \nu-24576 i \nu^{9} \mu^{2}-4212 i \mu \nu^{3}+20160 i \mu^{4} \nu+ \\
-1008 i \mu \nu+9600 i \nu^{5} \mu^{4}-6144 i \nu^{7} \mu^{3}-30528 i \nu^{3} \mu^{5}+11968 \nu^{8}-14016 i \nu^{5} \mu^{2}+16384 \nu^{1} 2+ \\
\left.-5376 \mu^{6}+16128 \mu^{5}+33920 \nu^{1} 0-5040 \mu^{2}-1497 \nu^{2}-8448 \nu^{4}\right) \\
\left.+2804 \nu^{6}-2736 \nu^{8}+2240 \mu^{6}-6720 \mu^{5}-2624 \nu^{10}+2100 \mu^{2}+670 \nu^{2}+3719 \nu^{4}\right) \\
+10048 \mu^{2} \nu^{8}-17280 \nu^{2} \mu^{5}+4032 \mu^{6} \nu^{4}-5600 \mu^{3}-420 \mu-24864 \nu^{6} \mu+37472 \nu^{6} \mu^{2}+35472 \nu^{4} \mu^{4}+ \\
-50784 \nu^{4} \mu^{3}-5720 \nu^{2} \mu+30880 \nu^{2} \mu^{4}-32960 \nu^{2} \mu^{3}+43844 \nu^{4} \mu^{2}+19320 \nu^{2} \mu^{2}-20468 \nu^{4} \mu+8400 \mu^{4}+ \\
\left(2 \mu^{2}-1\right)^{2}-4 \nu^{2} \\
2_{40}^{19}\left(\nu^{2}+1\right)^{2} \nu^{8} \\
+35-10048 \nu^{8} \mu-25216 \nu^{6} \mu^{3}+12608 \mu^{4} \nu^{6}+5760 \nu^{2} \mu^{6}-12096 \nu^{4} \mu^{5}+
\end{gathered}
$$

Acknowledgments: I thank Korea Institute for Advanced Study for providing computing resources (Abacus System) for this work. This work was initiated as a result of fruitfull discussions with M. Mazzocco.

\section{References}

[1] Y.V. Brezhnev: $\tau$-function solution of the Sixth Painlevé Transcendent. Theoretical and Mathematical Physics, 161(3), (2009), 1616 - 1633.

[2] A.D.Bruno, I.V. Goryuchkina: All the Asymptotic Expansions of Solutions of the Sixth Painlevé Equation. Doklady Mathematics, 76, (2007), 851- 855.

[3] A.D.Bruno, I.V. Goryuchkina: Asymptotic Expansions of Solutions of the Sixth Painlevé Equation. Moscow Math. Soc., S0077-1554(2010)00186-0, (2010), 1-104.

[4] B.Dubrovin: Geometry of 2D topological field theories, Lecture Notes in Math, 1620, (1996), 120-348.

[5] B. Dubrovin: Painlevé transcendents in to-dimensional topological field theory. In R. Conte (ed.): The Painlevé property, one century later. Springer, Ne York (1999). 
[6] B. Dubrovin, M. Mazzocco: Monodromy of certain Painlevé transcendents and reflection groups, Invent.Math., 141, (2000), 55-147.

[7] B. Dubrovin; M. Mazzocco: Canonical structure and symmetries of the Schlesinger equations. Comm. Math. Phys. 271 (2007), no. 2, 289373.

[8] D. Guzzetti: Stokes matrices and monodromy for the quantum cohomology of projective spaces Comm.Math.Phys 207, (1999), 341-383.

[9] D. Guzzetti: Inverse Problem and Monodromy Data for 3-dimensional Frobenius Manifolds . Mathematical Physics, Analysis and Geometry, 4, (2001), 254-291.

[10] D. Guzzetti On the Critical Behavior, the Connection Problem and the Elliptic Representation of a Painlevé 6 Equation. Mathematical Physics, Analysis and Geometry, 4, (2001), 293-377.

[11] D. Guzzetti: The Elliptic Representation of the General Painlevé VI Equation, Comm. Pure Appl. Math. LV, (2002), 1280-1363.

[12] D. Guzzetti: Matching procedure for the sixth Painlevé equation, J. Phys. A: Math. Gen. 39 (2006), 11973-1231

[13] D. Guzzetti: The Logaritmic asymptotics of the sixth Painlevé equation, J. Phys. A: Math. Theor. 41 (2008), 205201(46p)

[14] D. Guzzetti: Solving the sixth Painleve equation: towards the classification of all the critical behaviors and the connection formulae. International Mathematics Research Notices (2011); doi: 10.1093/imrn/rnr071

[15] D. Guzzetti: Tabulation of PVI transcendents and Parametrization Formulas. ArXiv:1108.3401 (2011).

[16] N. Hitchin: Twistor spaces, Einstein metrics and isometric deformations. J. Differential Geom., 42, (1995), 30-112

[17] K. Iwasaki: An area-preserving action of the modular group on cubic surfaces and the Painlevé VI equation, Comm.Math.Phys., 242, (2003) 185-219.

[18] K.Iwasaki, H.Kimura, S.Shimomura, M.Yoshida: From Gauss to Painleve'. Aspects of Mathematics 16, (1991).

[19] M. Jimbo: Monodromy Problem and the Boundary condition for some Painlevé Equations, Publ. RIMS, Kyoto Univ., 18, (1982), 1137-1161.

[20] M. Jimbo, T. Mia, K. Ueno: Monodromy Preserving Deformations of Linear Ordinary Differential Equations with Rational Coefficients (I). Physica D 2 , (1981), 306-352

[21] M.Mazzocco: Rational Solutions of the Painlevé VI Equation, J. Phys. A: Math. Gen. 34, (2001), 2281-2294.

[22] M.Mazzocco: Picard and Chazy solutions to the Painlevé VI equation, Math. Ann. 321 (2001), 157195. 
[23] K.Okamoto: Studies on the Painlevé Equation. I - Sixth Painlevé Equation $P_{V I}$, Ann. Mat. Pura Appl. 148, (1987), 337-381.

[24] Picard, E.: Mémoire sur la Théorie des functions algébriques de deux variables. Journal de Liouville, $\mathbf{5}$, (1889), 135- 319

[25] M. Sato, T. Mia, M. Jimbo: Holonomic Quantum Fields. II, Publ. RIMS, Kyoto Univ., 15, (1979), 201-278.

[26] S. Shimomura: A Family of Solutions of a Nonlinear Ordinary Differntial Equation and its Application to Painlevé Equations (III), (V), (VI). J.Math. Soc. Japan, 39, (1987), 649-662.

[27] H. Watanabe: Birational canonical transformations and classical solutions of the sixth Painlevé equation. Ann. Scuola Norm. Sup. Pisa Cl Sci. 27, (1999), 379-425. 